Udari Colombage ORCID iD: 0000-0003-0662-9363

Kuan-Yin Lin ORCID iD: 0000-0002-2902-3156

Helena Frawley ORCID iD: 0000-0002-7126-6979

\title{
Prevalence and impact of bladder and bowel disorders in women with breast cancer: a systematic review with meta-analysis
}

\author{
Udari N Colombage ${ }^{1}$, Kuan-Yin Lin ${ }^{2,3}$, Sze-Ee Soh ${ }^{1,4}$, Helena C Frawley ${ }^{1}$ \\ ${ }^{1}$ Department of Physiotherapy, Monash University, Melbourne, Australia. \\ ${ }^{2}$ Department of Physical Therapy, National Cheng Kung University, Tainan, Taiwan. \\ ${ }^{3}$ Institute of Allied Health Sciences, College of Medicine, National Cheng Kung \\ University, Tainan, Taiwan. \\ ${ }^{4}$ Department of Epidemiology and Preventive Medicine, Monash University, \\ Melbourne, Australia.
}

Short running title: Bladder \& bowel disorders in breast cancer.

Corresponding author: Udari Colombage, Monash University Peninsula Campus, 47 - 49 Moorooduc Highway, Frankston VIC 3199, Australia. +61404 867739.

Udari.Colombage@monash.edu

PROSPERO registration ID: CRD42018112100

Total word count: 2,675 without abstract or references.

This is the author manuscript accepted for publication and undergone full peer review but has not been through the copyediting, typesetting, pagination and proofreading process, which may lead to differences between this version and the Version of Record. Please cite this article as doi: 10.1002/nau.24531.

This article is protected by copyright. All rights reserved. 


\title{
Author contributions:
}

All authors contributed to the systematic review. Study selection was conducted by Udari Colombage and Kuan-Yin Lin. Data extraction and analysis were performed by Udari Colombage. All authors were involved in data interpretation. The first draft of the manuscript was written by Udari Colombage and all authors commented on previous versions of the manuscript. All authors read and approved the final manuscript.

\section{Conflict of interest disclosure:}

Miss Colombage has nothing to disclose. Dr Lin has nothing to disclose. Dr Soh has nothing to disclose. Associate Professor Frawley has nothing to disclose.

\begin{abstract}
Background: One of the consequences of breast cancer treatments may be the onset of new, or aggravation of pre-existing bladder and bowel disorders. However, the presence and impact of these disorders in women with breast cancer are poorly documented. The aim of this systematic review was to assess the prevalence, incidence and impact of bladder and bowel disorders in women undergoing breast cancer treatment. Methods: A systematic search of six databases was conducted. Pooled prevalence rates and impact of bladder and bowel disorders were calculated using random-effects models. Results: 32 studies met the inclusion criteria, and 17 studies were included in the meta-analyses. The pooled estimate of women who experienced bladder disorders following sensitivity analysis, which removed one study reporting a result that deviated from the pooled estimate, was 38\% [95\%CI 32$44 \%$; $\left.I^{2}=98 \% ; n=4,584\right]$. The impact of bladder and bowel disorders on women's daily lives was relatively low [bladder (scale $0-4$ ): mean 0.8; 95\%CI 0.4-1.1; $\mathrm{I}^{2}=99 \%$; This article is protected by copyright. All rights reserved.
\end{abstract}


$n=4,908$; bowel (scale 0-100): mean 14.2; 95\%CI 9.4-19; $\mathrm{I}^{2}=95 \%$; $\left.\mathrm{n}=1,024\right]$.

Conclusion: This is the first study to comprehensively document the magnitude of bladder and bowel disorders in the breast cancer population. This meta-analysis found that women with breast cancer had a higher prevalence of urinary incontinence (38\%) compared to women without breast cancer (21\%). Given the extent and impact of our findings, screening and management of bladder and bowel disorders may be indicated in women with breast cancer in order to improve their health-related quality of life.

Keywords: Bladder disorders, Bowel disorders, Breast cancer, Pelvic floor dysfunction.

\section{Introduction}

Breast cancer is the most common cancer affecting women ${ }^{1}$. The consequences of breast cancer treatments may impact on many aspects of physical and mental health ${ }^{2}$, including for some women, pelvic floor health ${ }^{3}$. This is presumed to be a result of ovarian suppression and failure secondary to chemotherapy in premenopausal women, and the use of endocrine therapy (including aromatase inhibitors and tamoxifen) in both pre- and postmenopausal women ${ }^{4}$. Prolonged effects of hypoestrogenism may be an etiological factor in the development of pelvic floor disorders, including bladder and bowel disorders ${ }^{5}$. In women who have undergone breast cancer treatments, the abrupt onset of symptoms attributed to menopause - vasomotor symptoms and sexual dysfunction - are well documented ${ }^{3}$. However, much less is known about bladder and bowel disorders and how these disorders impact on health-related quality of life (HRQoL) of women during and after their breast cancer treatment. While there is evidence of chemotherapy and endocrine therapy causing ovarian suppression ${ }^{4}$, whether the type of breast cancer or the type breast cancer treatment received is 
associated with the prevalence and impact of bladder and bowel disorders remains unknown.

A systematic review by Donovan et $\mathrm{al}^{4}$ investigated the prevalence of urinary disorders in women with breast cancer. Authors did not conduct a meta-analysis and an overall prevalence and impact of bladder disorders was not reported ${ }^{4}$. Pooled prevalence and impact data of bladder and bowel disorders will allow for the comparison of these disorders to women without breast cancer. This will improve our understanding of whether having breast cancer has an effect on the presence and impact of bladder and bowel disorders in women.

As further studies, representing double the number of women with breast cancer, have been published since 2012, an update to Donovan et $\mathrm{al}^{4}$ is warranted. Additionally, no systematic review to date has investigated bowel disorders in this population, representing a significant knowledge gap. Such data provide insight into the extent of this often over-looked problem in women with breast cancer. Therefore, the aim of this systematic review was to assess the prevalence, incidence and impact (severity or bother) of bladder and bowel disorders in women undergoing treatment for breast cancer.

Methods

Search and study selection

Relevant studies were identified through a search of six electronic databases (Ovid Medline, Ovid Embase, Ovid EmCare, Cumulative Index to Nursing and Allied Health Literature plus (CINAHL plus), PsychINFO and Scopus) from inception to December 2018. The Medical Subject Heading ${ }^{6}$ search terms used included "breast neoplasms”, “urogenital system” and "pelvic floor disorders” (See Appendix 1). The This article is protected by copyright. All rights reserved. 
search strategy was limited to studies published in English. Reference lists of identified articles were also hand-searched. Two reviewers (UC, KYL) independently applied the eligibility criteria to identified studies based on titles and abstracts, then full texts ${ }^{7}$. Any disagreements were resolved by a third reviewer ${ }^{7}(\mathrm{HF})$.

\section{Selection criteria}

Studies were included if it: (1) included women over 18 years old with breast cancer (stages 0-IV); (2) reported data on the prevalence and/or incidence, and/or impact of bladder and bowel disorders; and (3) full papers published in English. Studies had to report data on an identifiable and separately analysed group of women with breast cancer. If not, authors had to report that at least $75 \%$ of the full cohort represented women with breast cancer to be eligible for inclusion. Systematic reviews and qualitative studies were excluded.

\section{Data extraction}

One reviewer (UC) independently completed the data extraction using a customised and piloted data extraction table, following which a second reviewer (RA) crosschecked for accuracy. Extracted data included study characteristics, participant demographics, prevalence, incidence and impact of bladder and bowel disorders. Percentages or the number of women with bladder or bowel disorders were extracted to assess prevalence and incidence. Any type of questionnaire assessing the impact (severity or bother) of bladder and bowel disorders was accepted and the means and standard deviations of questionnaire scores were extracted. Impact was measured only in participants who reported the presence of bladder or bowel symptoms. Attempts were made to contact authors of studies for missing data.

This article is protected by copyright. All rights reserved. 
Risk of bias assessment

The risk of bias of all included studies was assessed using a customised risk of bias assessment tool (Appendix 2) which comprised items from the Joanna Briggs Institute's critical appraisal tool for prevalence studies ${ }^{8}$ and the Strengthening the Reporting of Observational studies in Epidemiology (STROBE) statement ${ }^{9}$. Two reviewers (UC, KYL) completed the risk of bias assessment independently by assessing all items of the checklist, scoring yes, no, or unclear. Any disagreements were resolved by a third reviewer ${ }^{7}(\mathrm{HF})$.

Synthesis of results

The pooled proportion of women experiencing bladder or bowel symptoms was calculated using random effects meta-analysis. Variances of the raw proportions were transformed using a Freeman-Tukey arcsine square root transformation so that confidence intervals of proportions became asymmetrical and did not exceed the range of 0 to $1^{10}$. A DerSimonian-Laird random effects model was used to pool the transformed proportions, which incorporated a measure of the extent of betweenstudy heterogeneity ${ }^{10}$. A random effects model was used to pool impact scores of bladder and bowel disorders in women with breast cancer when $\mathrm{I}^{2}$ was greater than $50 \%$, indicating statistical heterogeneity ${ }^{10}$. Data were pooled in a meta-analysis when at least four studies reported on either the number of women experiencing bladder or bowel disorders, or the impact of these disorders ${ }^{11}$. Where data were unable to be pooled in a meta-analysis, a descriptive analysis was performed ${ }^{23}$. Sensitivity analyses were conducted to determine the robustness of prevalence and impact outcomes where outliers were detected ${ }^{12}$. Additional subgroup analyses for the prevalence and impact of specific bladder and bowel disorders as well as breast cancer treatments, stage and time since diagnosis were conducted where possible in order to identify

This article is protected by copyright. All rights reserved. 
sub-groups of women with breast cancer who were most at risk and impacted by bladder and bowel disorders. All meta-analyses were performed using Stata v16.0 (StataCorp, LLC).

Results

Search yield

A total of 3,405 articles were identified, 32 studies were included in the qualitative synthesis, and 17 studies were included in the meta-analysis (see Figure 1). Our review included seven randomised controlled trials ${ }^{13,14,15,16,17,18,19}$, eight cohort studies $^{20,21,22,2,23,24,25,26}$ and 17 cross-sectional studies $^{27,28,29,30,31,32,33,34,35,36,37,38,39,40,41,42,43}$. A total of 12,611 women with breast cancer were represented in this systematic review; 10,441 of these women were in the meta-analysis.

$<$ Insert Figure 1 here $>$

Figure 1: PRISMA flowchart of study selection process

This article is protected by copyright. All rights reserved. 


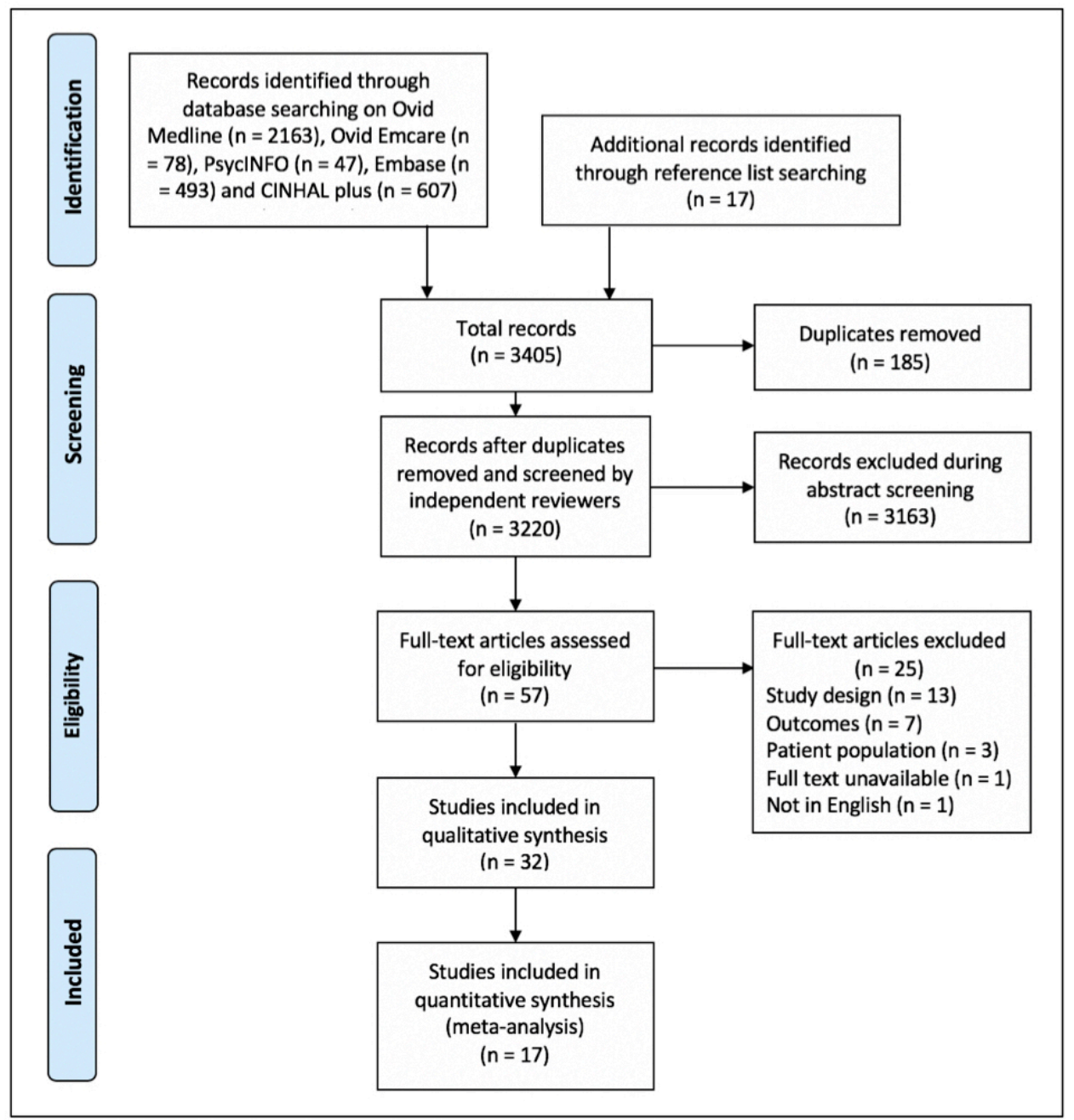

Figure 1: PRISMA flowchart of study selection process

Risk of bias assessment

A summary of the risk of bias assessment is presented in Table 1. The risk of bias for RCTs were considered low, and moderate for cohort and cross-sectional studies. Only three studies ${ }^{10,24,25}$ used outcome measures validated for the purpose of assessing the prevalence and impact of bladder and bowel disorders. A number of studies 13,19,20,22,24 $^{2}$ were unclear regarding the sampling frame included in the study, especially around breast cancer stage, treatment type or the number of years since breast cancer diagnosis. Furthermore, most studies ${ }^{10,11,12,13,14,17,18,20,22,28,31,32,33,34,36,40}$ did not

This article is protected by copyright. All rights reserved. 
explicitly state the statistical analysis method for assessing the prevalence and impact of bladder and bowel disorders.

Table 1: Risk of bias assessment

$<$ Insert Table 1 here >

Y, yes; N, no; U, unclear; NA, not applicable.

Demographic and clinical characteristics of included studies

The characteristics of the included studies is shown in Table 2. The mean age across all studies was 54.3 years, and sample sizes ranged from 35 to 2,208 participants. Most studies collected data during or shortly after primary cancer treatment. In studies that reported follow-up time points, the median time post-diagnosis when participants were assessed was two years (range 1 month-5 years) ) $^{2,20,13,18,24,25,29,36,38,40,42}$. Of the studies that provided a range of follow-up time-points, all were within five years post $\operatorname{diagnosis}^{14,32,37,39,43}$, except two studies ${ }^{27,35}$ which followed-up for 10 years. Fourteen studies did not indicate the number of years post diagnosis $^{15,19,21,26,28,30,31,33,17,22,16,23,34,41}$. A detailed description of the outcome measures used in included studies have been summarised in Appendix 3.

Table 2: Characteristics of included studies

$\alpha$ : Authors provided proportions of women in age range.

$ß:$ Authors provided mean age for each sample. Sample 1: 56, Sample 2: 50, Sample 3: 57.

$\gamma$ : Authors provided staging for each group. Sample 1: 0-II; Sample 2: 0-II; Sample 3: I-II.

This article is protected by copyright. All rights reserved. 
NR, not reported; DCIS, ductal carcinoma in situ; Chemo, chemotherapy; Radio, radiation therapy; Endo, endocrine therapy; EORTC QLQ-C30, European Organization for the Research and Treatment of Cancer Quality of Life Questionnaire; MRS, Menopause Rating Scale; BCPT, Breast Cancer Prevention Trial; CARES, Cancer Rehabilitation Evaluation System; UDI-6, urinary distress inventory; FACT, Functional Assessment of Cancer Therapy; BFLUTS, Bristol Female Lower Urinary Tract Symptoms questionnaire; GCS, Greene Climacteric Scale; QUID, Questionnaire for Urinary Incontinence Diagnosis; VAS, Visual Analog Scale.

\section{Prevalence of bladder disorders}

The pooled estimate of the proportion of women with breast cancer experiencing any type of bladder disorder was 0.33 [95\%CI 0.24-0.42; $\mathrm{I}^{2}=99 \%$; $\left.\mathrm{n}=5,384\right]$ (see Figure 2). As two studies ${ }^{20,39}$ reported different data values for stress UI, the value most closely aligned with accepted definitions of stress $\mathrm{UI}^{44}$ was extracted. Subgroup analyses according to breast cancer treatment type, stage, and time since diagnosis were not conducted as these details were not reported by the included studies.

$<$ Insert Figure 2 here $>$

Figure 2: Meta-analysis of the proportion of women reporting any bladder disorders

This article is protected by copyright. All rights reserved. 


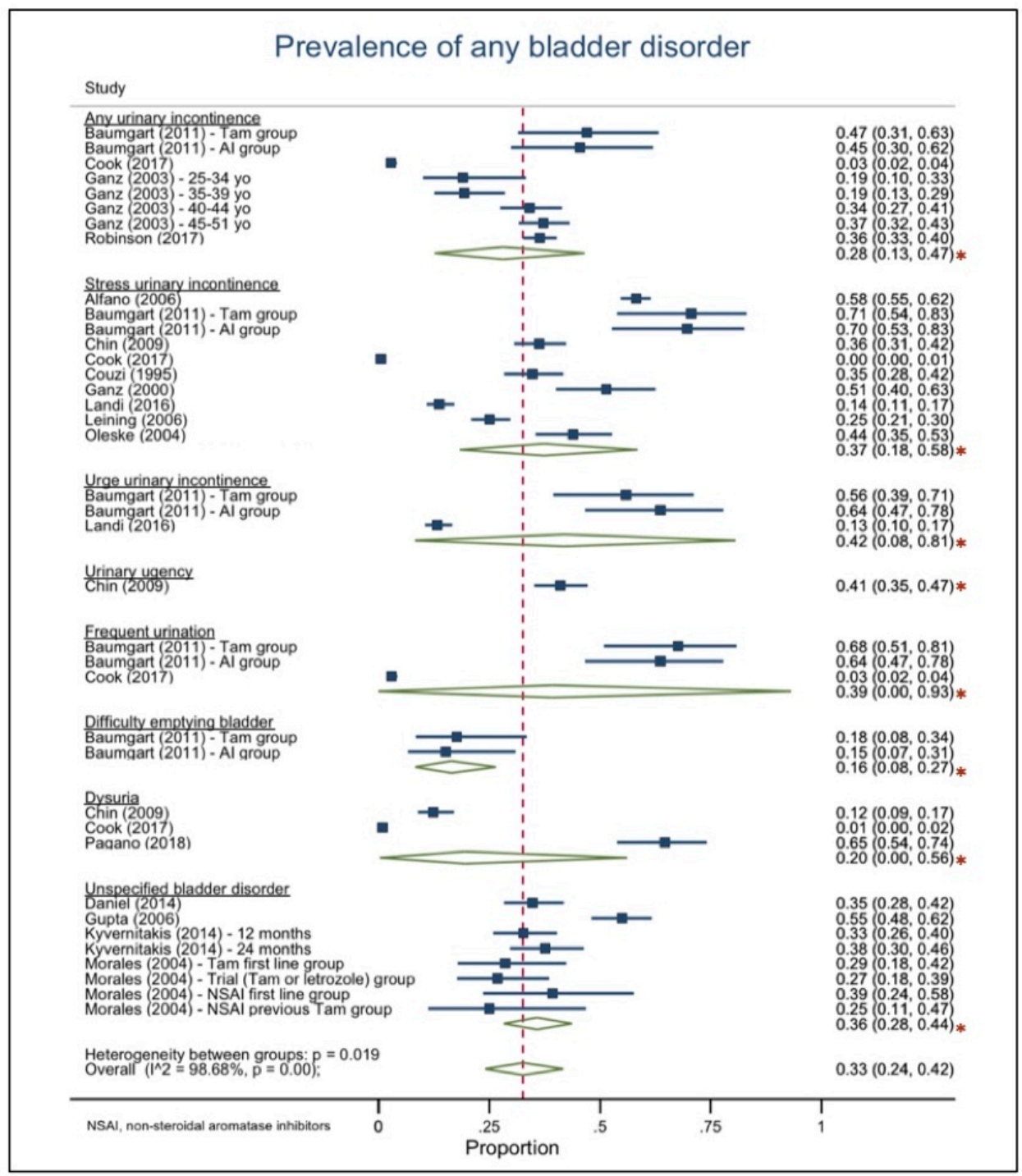

Figure 2: Meta-analysis of the proportion of women reporting any bladder disorders

* Asterisks indicate the pooled prevalence estimates for each bladder or bowel disorder type.

* Asterisks indicate the pooled prevalence estimates for each type of bladder disorder. The prevalence rate reported in Cook et $\mathrm{al}^{32}$ appeared to deviate from the pooled estimate, most likely as the authors performed a retrospective search of medical records for the presence of bladder disorders. When this study was excluded from the analysis (not shown), the pooled estimate of the proportion of women with breast

This article is protected by copyright. All rights reserved. 
cancer experiencing bladder disorders increased from 0.33 to 0.38 [95\%CI 0.32-0.44; $\left.\mathrm{I}^{2}=98 \% ; \mathrm{n}=4,584\right]$.

Subgroup analysis showed the pooled estimate of the proportion of women with breast cancer experiencing UI was 0.36 [95\%CI $\left.0.24-0.48 ; I^{2}=99 \% ; n=4,562\right]$. Following sensitivity analysis, the estimate increased slightly to 0.38 [95\%CI 0.30 $\left.0.48 ; I^{2}=99 \% ; n=3,762\right]$.

Prevalence of bowel disorders

Despite a number of studies commenting on the impact of bowel disorders on women with breast cancer ${ }^{17,19,24,30}$, only one study presented prevalence rates of bowel disorders $^{27}$. This study reported that $18 \%$ of women with breast cancer experienced faecal incontinence $(\mathrm{FI})^{27}$.

\section{Impact of bladder and bowel disorders}

The impacts of bladder and bowel disorders were documented in two different ways. Studies reporting the impact of bladder disorders commented on the severity of bother of bladder disorders, while studies reporting the impact of bowel disorders reported on the severity of bowel disorders.

Severity of bother of bladder disorders

Studies measuring the severity of bother of bladder disorders using the Breast Cancer Prevention Trial (BCPT) symptom checklist were pooled. The pooled mean estimate was 0.8 [95\%CI $\left.0.5-1.1 ; \mathrm{I}^{2}=100 \% ; \mathrm{n}=4,908\right]$, corresponding to slight bother (see Figure 3A). The pooled severity of bother of stress UI was 0.7 [95\%CI $0.3-1$; $\mathrm{I}^{2}=99 \%$; $n=4,173$ ] corresponding to slight bother (see Figure 3A). A sensitivity analysis did not result in a change to the overall pooled estimate, nor the corresponding bother rating.

This article is protected by copyright. All rights reserved. 
No meta-analyses were undertaken for studies reporting the severity of bother of other bladder disorders as there were either too few studies, or variance in the outcome measures used (summarised in Appendix 4).

Severity of bowel disorders

Four studies ${ }^{17,19,24,30}$ measured the severity of constipation and diarrhoea using the European Organisation for Research and Treatment of Cancer Quality of Life Questionnaire (EORTC QLQ-C30) (see Figure 3B). The pooled mean estimate of the severity of bowel disorders was 14.2 [95\%CI 9.4-18.9; $\mathrm{I}^{2}=95 \%$; $\mathrm{n}=1,024$ ] which corresponds to low severity. Sub-group analysis showed that the pooled mean estimate of the severity of constipation was 17.8 [95\%CI $12.1-23.5 ; \mathrm{I}^{2}=95 \%$; $\mathrm{n}=1,024]$, and diarrhoea was 10.5 [95\%CI 4.9-16.1; $\left.\mathrm{I}^{2}=95 \% ; \mathrm{n}=1,024\right]$. Both correspond to low severity. Sensitivity analysis did not result in a difference to the overall pooled estimate, nor to the severity of the bowel symptoms.

$<$ Insert Figure 3 here $>$

Figure 3: A) Meta-analysis of the impact of bladder disorders as measured by the BCPT symptom checklist, B) Meta-analysis of the impact of bowel disorders as measured by the EORTC QLQ-C30

This article is protected by copyright. All rights reserved. 
Discussion

This review identified an overall prevalence of bladder disorders in women with breast cancer of 38\%. One study reported a prevalence of faecal incontinence of $18 \%{ }^{27}$. Despite these data suggesting substantial prevalence, bladder and bowel disorders were rated as having low impact on the lives of women at short and medium-term follow-up of breast cancer treatment.

Following sensitivity analysis, our meta-analysis showed a pooled estimate of $38 \%$ of women with breast cancer experiencing UI. Given that $25-45 \%$ of women without breast cancer experience $\mathrm{UI}^{45}$, our findings suggests that the prevalence of $\mathrm{UI}$ in this population is similar to women without cancer. However, prevalence data of UI in women without cancer have been established from studies that drew from a range of prevalence frequencies from 'currently' to have 'ever' leaked ${ }^{45}$. Comparatively, women with breast cancer were asked to report presence of UI if they experienced leakage in the past four weeks, a stricter definition. When we compare the prevalence of UI using the same definition and age group, we find that women with breast cancer may have a higher prevalence (38\%) compared to women without breast cancer $\left(21 \%^{46}\right)$. Similarly, the single study reporting the prevalence of FI in women with breast cancer $\left(18 \%{ }^{27}\right)$ is higher than in women without breast cancer $\left(8-10 \%{ }^{47}\right)$. This is the first study to document this comparative finding and indicates that that the extent of these disorders in the breast cancer population is higher than previously acknowledged. This result has implications for screening and management of these symptoms in women with breast cancer who are bothered by their symptoms.

We found the level of bother or severity of bladder and bowel symptoms to be slight in women with breast cancer, which is consistent with women without breast cancer ${ }^{48}$.

This article is protected by copyright. All rights reserved. 
However, it is quite feasible that in the context of cancer diagnosis, treatment and treatment recovery, the impact of a non-fatal condition such as a bladder or bowel disorder may be rated comparatively lower ${ }^{49}$. In this review, most studies collected data during or shortly after primary cancer treatment, using cancer-specific questionnaires. The five-year survivorship point post-diagnosis is a milestone many women with breast cancer anticipate and health conditions unrelated to their cancer may be of lower priority during this time. Breast cancer survivors tend to seek information and treatment on their 'unmet needs', including menopausal symptoms caused by breast cancer treatments, once they have met their survivorship milestone ${ }^{50}$. As most studies in this review assessed the impact of bladder and bowel disorders before this five-year milestone, it is possible that women prioritised cancer-related symptoms, and under-reported the impact of other symptoms. The two studies which followed women for 10 years did not report the impact of bladder and bowel disorders $^{27,35}$. Therefore, the longer-term impact of bladder and bowel disorders in women with breast cancer, and whether this impact changes over time, remains unknown.

None of our included studies reported the incidence of bladder and bowel disorders. This is important to acknowledge as women included in these studies may have had pre-existing bladder and bowel disorders, before their breast cancer diagnosis. Therefore, we are unable to determine whether receiving treatment for breast cancer has any effect on the presence or impact of bladder and bowel disorders. It is also possible that women may not be interested to seek treatment for bladder and bowel disorders if these conditions pre-dated the cancer diagnosis.

This article is protected by copyright. All rights reserved. 
Since the previous published systematic review ${ }^{4}$, we have included 17 additional articles representing double the number of women with breast cancer (6,397 women in Donovan et $\mathrm{al}^{4} ; 12,611$ women in this study). We were also able to conduct metaanalyses which provided us with pooled prevalence rates and impact scores to understand the magnitude and burden of bladder and bowel symptoms in women with breast cancer compared to women without breast cancer. This is also the first systematic review to document bowel disorders in women with breast cancer.

\section{Limitations of research}

Our search strategy was limited to studies in English and studies in other languages may have been missed. The majority of studies included were cross-sectional in nature. Whilst causal inferences cannot be drawn from our findings, this is the first study to pool prevalence rates in women with breast cancer. Finally, studies included women at various time-points along their treatment trajectory, however the prevalence or impact of bladder and bowel disorders according to breast cancer stage, treatment type, or duration of time on each treatment were not specified. We were therefore unable to complete any sub-group analyses according to these characteristics which would have provided information on identifying sub-groups of women with breast cancer who were perhaps most at risk of bladder and bowel disorders. As such, our findings may not be generalised to specific sub-groups of women with breast cancer (e.g. women on specific endocrine therapies such as tamoxifen or aromatase inhibitors). Future studies should attempt to stratify analyses according to breast cancer stage, treatment type and time since breast cancer diagnosis.

This article is protected by copyright. All rights reserved. 


\section{Conclusion}

This is the first study to comprehensively document the magnitude of bladder and bowel disorders in the breast cancer population. This meta-analysis found that women with breast cancer had a higher prevalence of urinary incontinence (38\%) compared to women without breast cancer $\left(21 \%{ }^{46}\right)$. Given the extent and impact of our findings, screening and management of bladder and bowel disorders may be indicated in women with breast cancer in order to improve their health-related quality of life.

Appendix 1: Search strategy

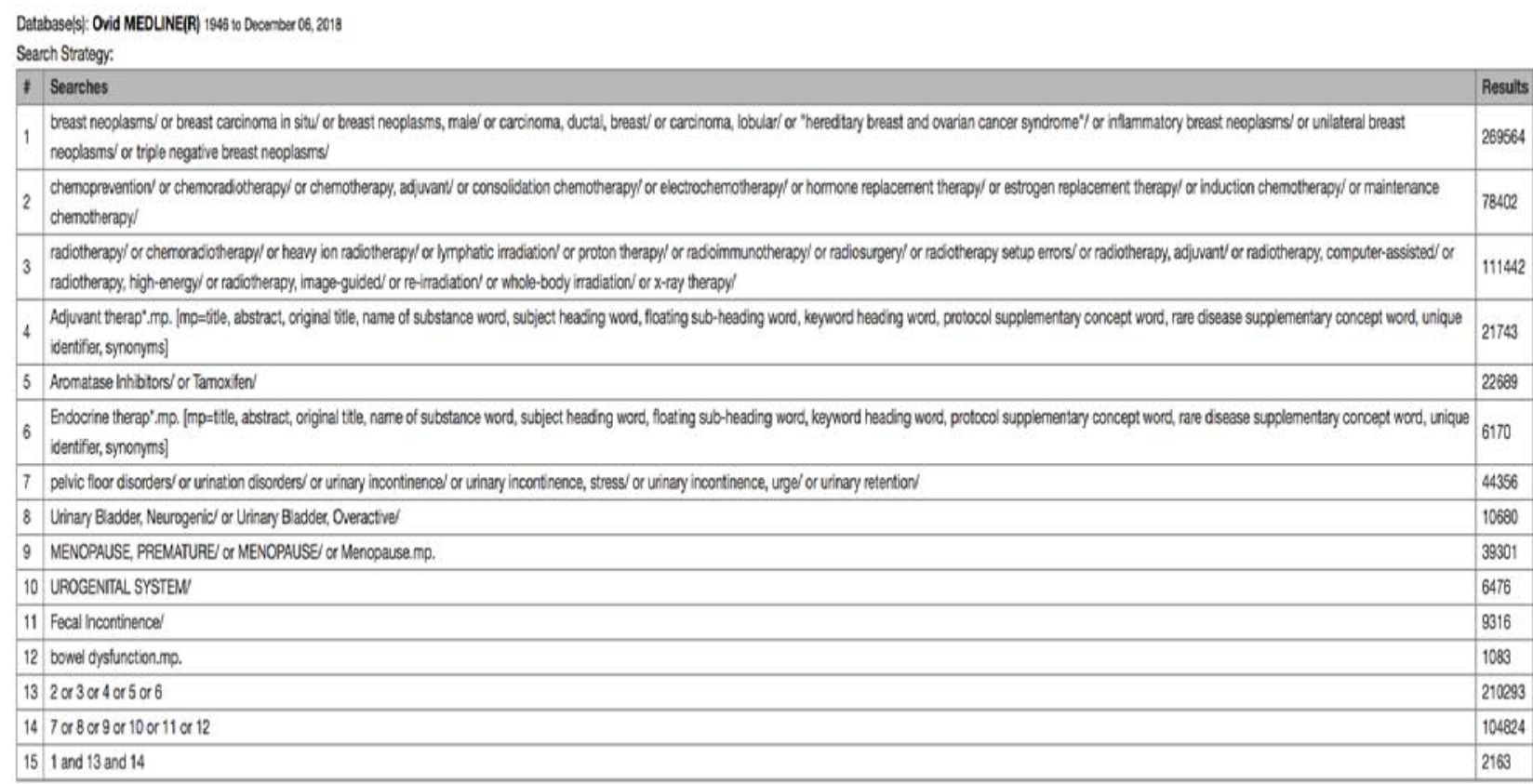

Appendix 2: Customised risk of bias assessment tool

\section{Sample characteristics}

1. Were study participants sampled in an appropriate way?

2. Was the sample frame appropriate to address the target population?

3. Were the inclusion criteria defined?

4. Were the study subjects and the setting described in detail?

5. Was the sample size adequate?

\section{Confounding}

This article is protected by copyright. All rights reserved. 
6. Were confounding factors identified?

7. Were strategies for dealing with confounding factors stated?

\section{Outcomes and analysis}

8. Was the outcome (prevalence or impact of bladder/bowel conditions) measured in a valid and reliable way for all participants?

9. Was there appropriate statistical analysis for analysis of prevalence or impact of bladder/bowel conditions?

10. Were the reasons for incomplete follow up or missing data described?

11. Was the response rate adequate, and if not, was the low response rate managed appropriately?

12. Were key findings stated?

13. Were limitations of the study described, taking into account sources of potential bias?

\section{Assessing risk of bias}

Number of Yes's:

- $0-4=$ High risk of bias

- 5-9 = Moderate risk of bias

- $10-13$ = Low risk of bias

Appendix 3: Outcome measures used in included studies to assess the presence or impact of bladder and bowel disorders.

\begin{tabular}{|c|c|c|c|c|}
\hline $\begin{array}{c}\text { Author } \\
\text { (year) }\end{array}$ & $\begin{array}{l}\text { Measurement } \\
\text { tool used }\end{array}$ & Question wording & $\begin{array}{c}\text { Validated } \\
\text { questionnaire }\end{array}$ & $\begin{array}{l}\text { Validated for } \\
\text { the purpose of } \\
\text { assessing the } \\
\text { presence or } \\
\text { impact of } \\
\text { bladder/bowel } \\
\text { disorders }\end{array}$ \\
\hline $\begin{array}{l}\text { Duijts } \\
(2012)^{10}\end{array}$ & BFLUTS & $\begin{array}{l}\text { Does urine leak before } \\
\text { you can get to the } \\
\text { toilet? }\end{array}$ & Yes & Yes \\
\hline $\begin{array}{l}\text { Ganz } \\
(2000)^{11}\end{array}$ & $\begin{array}{l}\text { Menopause } \\
\text { symptom } \\
\text { scale from } \\
\text { BCPT } \\
\text { symptom } \\
\text { checklist }\end{array}$ & $\begin{array}{l}\text { How bothered have you } \\
\text { been by difficulty with } \\
\text { bladder control in the } \\
\text { past } 4 \text { weeks. } \\
\text { - SUI: difficulty } \\
\text { with bladder }\end{array}$ & Yes & No \\
\hline
\end{tabular}

This article is protected by copyright. All rights reserved. 


\begin{tabular}{|c|c|c|c|c|}
\hline & & $\begin{array}{l}\text { control at other } \\
\text { times (coughing, } \\
\text { sneezing) }\end{array}$ & & \\
\hline $\begin{array}{l}\text { Ganz } \\
(2016)^{12}\end{array}$ & $\begin{array}{l}\text { BCPT } \\
\text { symptom } \\
\text { checklist }\end{array}$ & $\begin{array}{l}\text { How bothered have you } \\
\text { been by difficulty with } \\
\text { bladder control in the } \\
\text { past } 4 \text { weeks. } \\
\text { - SUI: difficulty } \\
\text { with bladder } \\
\text { control at other } \\
\text { times (coughing, } \\
\text { sneezing) }\end{array}$ & Yes & No \\
\hline $\begin{array}{l}\text { Land } \\
(2004)^{13}\end{array}$ & $\begin{array}{l}\text { Selected } \\
\text { questions } \\
\text { from BCPT } \\
\text { symptom } \\
\text { checklist }\end{array}$ & $\begin{array}{l}\text { How bothered have you } \\
\text { been by difficulty with } \\
\text { bladder control in the } \\
\text { past } 4 \text { weeks. } \\
\text { - SUI: difficulty } \\
\text { with bladder } \\
\text { control at other } \\
\text { times (coughing, } \\
\text { sneezing) }\end{array}$ & Yes & No \\
\hline
\end{tabular}

Appendix 3: Outcome measures used in included studies to assess the presence or impact of bladder and bowel disorders (continued)

\begin{tabular}{|l|l|l|c|c|}
\hline $\begin{array}{c}\text { Author } \\
\text { (year) }\end{array}$ & $\begin{array}{c}\text { Measurement } \\
\text { tool used }\end{array}$ & Question wording & $\begin{array}{c}\text { Validated } \\
\text { questionnaire }\end{array}$ & $\begin{array}{c}\text { Validated for } \\
\text { the purpose of } \\
\text { assessing the } \\
\text { presence or } \\
\text { impact of } \\
\text { bladder/bowel } \\
\text { disorders }\end{array}$ \\
\hline $\begin{array}{l}\text { Lötzke } \\
(2016)^{14}\end{array}$ & $\begin{array}{l}\text { EORTC } \\
\text { QLQ-C30 }\end{array}$ & $\begin{array}{l}\text { Have you been } \\
\text { constipated? Have } \\
\text { you had diarrhea? }\end{array}$ & Yes & No \\
\hline
\end{tabular}

This article is protected by copyright. All rights reserved. 


\begin{tabular}{|c|c|c|c|c|}
\hline $\begin{array}{l}\text { Schover } \\
(2006)^{15}\end{array}$ & $\begin{array}{l}\text { Menopausal } \\
\text { Symptom } \\
\text { Scale }\end{array}$ & $\begin{array}{l}\text { Please indicate the } \\
\text { extent to which you } \\
\text { are bothered by any } \\
\text { of these symptoms: } \\
\text { - SUI: } \\
\text { difficulty } \\
\text { with bladder } \\
\text { control while } \\
\text { laughing or } \\
\text { crying and } \\
\text { difficulty } \\
\text { with bladder } \\
\text { control at } \\
\text { other times. }\end{array}$ & Yes & No \\
\hline $\begin{array}{l}\text { Versmessen } \\
(2012)^{16}\end{array}$ & $\begin{array}{l}\text { EORTC } \\
\text { QLQ-C30 }\end{array}$ & $\begin{array}{l}\text { Have you been } \\
\text { constipated? Have } \\
\text { you had diarrhea? }\end{array}$ & Yes & No \\
\hline $\begin{array}{l}\text { Alfano } \\
(2006)^{17}\end{array}$ & $\begin{array}{l}\text { BCPT } \\
\text { symptom } \\
\text { checklist }\end{array}$ & $\begin{array}{l}\text { How bothered have } \\
\text { you been by } \\
\text { difficulty with } \\
\text { bladder control in } \\
\text { the past } 4 \text { weeks. } \\
\text { - SUI: } \\
\text { difficulty } \\
\text { with bladder } \\
\text { control at } \\
\text { other times } \\
\text { (coughing, } \\
\text { sneezing) }\end{array}$ & Yes & No \\
\hline $\begin{array}{l}\text { Biglia } \\
(2010)^{18}\end{array}$ & GCS & $\begin{array}{l}\text { Indicate the extent } \\
\text { to which you are } \\
\text { bothered at the } \\
\text { moment by } \\
\text { urogenital } \\
\text { symptoms. }\end{array}$ & Yes & No \\
\hline $\begin{array}{l}\text { Hartl } \\
(2010)^{21}\end{array}$ & $\begin{array}{l}\text { EORTC } \\
\text { QLQ-C30 }\end{array}$ & $\begin{array}{l}\text { Have you been } \\
\text { constipated? Have } \\
\text { you had diarrhea? }\end{array}$ & Yes & No \\
\hline
\end{tabular}

This article is protected by copyright. All rights reserved. 
Appendix 3: Outcome measures used in included studies to assess the presence or impact of bladder and bowel disorders (continued)

\begin{tabular}{|c|c|c|c|c|}
\hline $\begin{array}{l}\text { Author } \\
\text { (year) }\end{array}$ & $\begin{array}{l}\text { Measurement } \\
\text { tool used }\end{array}$ & Question wording & $\begin{array}{c}\text { Validated } \\
\text { questionnaire }\end{array}$ & $\begin{array}{l}\text { Validated for } \\
\text { the purpose of } \\
\text { assessing the } \\
\text { presence or } \\
\text { impact of } \\
\text { bladder/bowel } \\
\text { disorders }\end{array}$ \\
\hline $\begin{array}{l}\text { Kyvernitakis } \\
(2014)^{19}\end{array}$ & $\begin{array}{l}\text { Menopausal } \\
\text { Rating Scale }\end{array}$ & $\begin{array}{l}\text { Which of the } \\
\text { following } \\
\text { symptoms apply to } \\
\text { you at this time? } \\
\text { Bladder problems } \\
\text { (difficulty in } \\
\text { urinating, increased } \\
\text { need to urinate, } \\
\text { bladder } \\
\text { incontinence) }\end{array}$ & Yes & No \\
\hline $\begin{array}{l}\text { Ganz } \\
(2011)^{2}\end{array}$ & $\begin{array}{l}\text { BCPT } \\
\text { symptom } \\
\text { checklist }\end{array}$ & $\begin{array}{l}\text { How bothered have } \\
\text { you been by } \\
\text { difficulty with } \\
\text { bladder control in } \\
\text { the past } 4 \text { weeks. } \\
\text { - SUI: } \\
\text { difficulty } \\
\text { with } \\
\text { bladder } \\
\text { control at } \\
\text { other times } \\
\text { (coughing, } \\
\text { sneezing) }\end{array}$ & Yes & No \\
\hline $\begin{array}{l}\text { Morales } \\
(2004)^{23}\end{array}$ & $\begin{array}{l}\text { Author- } \\
\text { designed } \\
\text { questionnaire }\end{array}$ & $\begin{array}{l}\text { Did you have } \\
\text { urinary problems? }\end{array}$ & Yes & No \\
\hline $\begin{array}{l}\text { Oleske } \\
(2004)^{22}\end{array}$ & $\begin{array}{l}\text { Symptom } \\
\text { Rating Scale } \\
\text { from BCPT } \\
\text { symptom }\end{array}$ & $\begin{array}{l}\text { How bothered have } \\
\text { you been by } \\
\text { difficulty with } \\
\text { bladder control in }\end{array}$ & Yes & No \\
\hline
\end{tabular}

This article is protected by copyright. All rights reserved. 


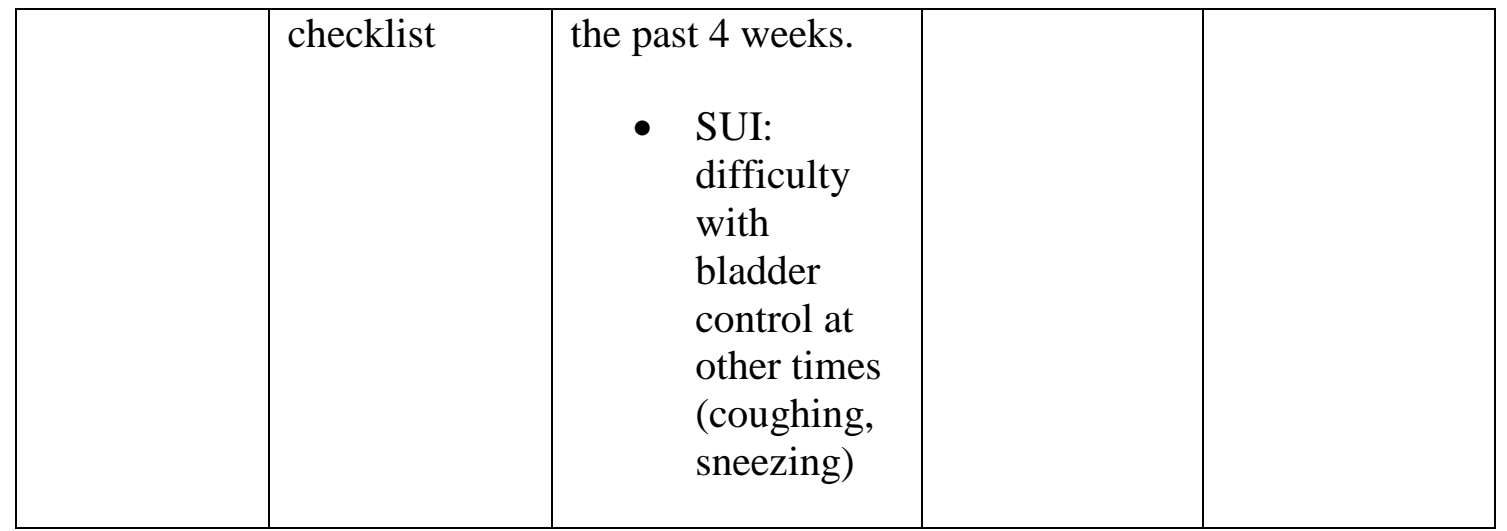

Appendix 3: Outcome measures used in included studies to assess the presence or impact of bladder and bowel disorders (continued)

\begin{tabular}{|c|c|c|c|c|}
\hline $\begin{array}{c}\text { Author } \\
\text { (year) }\end{array}$ & $\begin{array}{l}\text { Measurement } \\
\text { tool used }\end{array}$ & Question wording & $\begin{array}{c}\text { Validated } \\
\text { questionnaire }\end{array}$ & $\begin{array}{l}\text { Validated for } \\
\text { the purpose of } \\
\text { assessing the } \\
\text { presence or } \\
\text { impact of } \\
\text { bladder/bowel } \\
\text { disorders }\end{array}$ \\
\hline $\begin{array}{l}\text { Pagano } \\
(2018)^{20}\end{array}$ & $\begin{array}{l}\text { Visual } \\
\text { analogue scale }\end{array}$ & $\begin{array}{l}\text { Participants were } \\
\text { assessed for dysuria } \\
\text { (pain on urination) by } \\
\text { rating on visual } \\
\text { analogue scale }\end{array}$ & Yes & No \\
\hline $\begin{array}{l}\text { Avis } \\
(2004)^{26}\end{array}$ & CARES & $\begin{array}{l}\text { To what extent are } \\
\text { you bothered by any } \\
\text { of these symptoms - } \\
\text { difficulty with bladder } \\
\text { control while laughing } \\
\text { or crying and } \\
\text { difficulty with bladder } \\
\text { control at other times. }\end{array}$ & Yes & No \\
\hline $\begin{array}{l}\text { Baumgart } \\
(2011)^{25}\end{array}$ & UDI-6 & $\begin{array}{l}\text { Do you experience } \\
\text { and, if so, how much } \\
\text { are you bothered by: } \\
\text { Frequent Urination? } \\
\text { Urine leakage related }\end{array}$ & Yes & Yes \\
\hline
\end{tabular}

This article is protected by copyright. All rights reserved. 


\begin{tabular}{|c|c|c|c|c|}
\hline & & $\begin{array}{l}\text { to urgency? } \\
\text { Urine leakage related } \\
\text { to physical activity } \\
\text { (walking, running, } \\
\text { laughing, sneezing, } \\
\text { coughing)? } \\
\text { Difficulty completely } \\
\text { emptying bladder? }\end{array}$ & & \\
\hline $\begin{array}{l}\text { Chen } \\
(2018)^{27}\end{array}$ & $\begin{array}{l}\text { EORTC } \\
\text { QLQ-C30 }\end{array}$ & $\begin{array}{l}\text { Have you been } \\
\text { constipated? Have you } \\
\text { had diarrhea? }\end{array}$ & Yes & No \\
\hline $\begin{array}{l}\text { Chin } \\
(2009)^{28}\end{array}$ & $\begin{array}{l}\text { Author- } \\
\text { designed } \\
\text { questions }\end{array}$ & $\begin{array}{l}\text { Author-designed } \\
\text { questions relating to } \\
\text { urinary incontinence, } \\
\text { dysuria and urgency. }\end{array}$ & No & No \\
\hline
\end{tabular}

Appendix 3: Outcome measures used in included studies to assess the presence or impact of bladder and bowel disorders (continued)

\begin{tabular}{|l|l|l|c|c|}
\hline $\begin{array}{c}\text { Author } \\
\text { (year) }\end{array}$ & $\begin{array}{c}\text { Measurement } \\
\text { tool used }\end{array}$ & Question wording & $\begin{array}{c}\text { Validated } \\
\text { questionnaire }\end{array}$ & $\begin{array}{c}\text { Validated for } \\
\text { the purpose of } \\
\text { assessing the } \\
\text { presence or } \\
\text { impact of } \\
\text { bladder/bowel } \\
\text { disorders }\end{array}$ \\
\hline $\begin{array}{l}\text { Cook } \\
(2017)^{29}\end{array}$ & NA & $\begin{array}{l}\text { Authors } \\
\text { retrospectively } \\
\text { searched medical } \\
\text { records. }\end{array}$ & NA & NA \\
\hline $\begin{array}{l}\text { Couzi } \\
(1995)^{30}\end{array}$ & $\begin{array}{l}\text { Author- } \\
\text { designed }\end{array}$ & $\begin{array}{l}\text { Author-designed } \\
\text { questionnaire } \\
\text { assessing the presence } \\
\text { and bother of } \\
\text { difficulty with bladder } \\
\text { control. }\end{array}$ & No & No \\
\hline Daniel & Menopause & $\begin{array}{l}\text { Which of the } \\
\text { following apply to }\end{array}$ & Yes & No \\
\hline
\end{tabular}

This article is protected by copyright. All rights reserved. 


\begin{tabular}{|l|l|l|l|l|}
\hline (2014) $^{31}$ & Rating Scale & $\begin{array}{l}\text { you at this time? } \\
\text { Bladder problems } \\
\text { (difficulty in } \\
\text { urinating, } \\
\text { increased need to } \\
\text { urinate, bladder } \\
\text { incontinence) }\end{array}$ & No \\
\hline $\begin{array}{l}\text { Ganz } \\
(2003)^{32}\end{array}$ & $\begin{array}{l}\text { BCPT } \\
\text { symptom } \\
\text { checklist }\end{array}$ & $\begin{array}{l}\text { How bothered have } \\
\text { you been by difficulty } \\
\text { with bladder control } \\
\text { in the past 4 weeks. }\end{array}$ & Yes & Nos \\
\hline $\begin{array}{l}\text { Greendale } \\
(2001)^{33}\end{array}$ & $\begin{array}{l}\text { BCPT } \\
\text { symptom } \\
\text { checklist }\end{array}$ & $\begin{array}{l}\text { How bothered have } \\
\text { you been by difficulty } \\
\text { with bladder control } \\
\text { in the past 4 weeks. }\end{array}$ & Yes & No \\
\hline $\begin{array}{l}\text { Gupta } \\
(2006)^{34}\end{array}$ & $\begin{array}{l}\text { Mating Scale } \\
\text { Renopause }\end{array}$ & $\begin{array}{l}\text { Which of the } \\
\text { following apply to } \\
\text { you at this time? } \\
\text { Bladder problems } \\
\text { (difficulty in } \\
\text { urinating, } \\
\text { increased need to } \\
\text { urinate, bladder } \\
\text { incontinence) }\end{array}$ & No & \\
\hline
\end{tabular}

Appendix 3: Outcome measures used in included studies to assess the presence or impact of bladder and bowel disorders (continued)

\begin{tabular}{|c|c|c|c|c|}
\hline $\begin{array}{c}\text { Author } \\
\text { (year) }\end{array}$ & $\begin{array}{c}\text { Measurement } \\
\text { tool used }\end{array}$ & Question wording & $\begin{array}{c}\text { Validated } \\
\text { questionnaire }\end{array}$ & $\begin{array}{c}\text { Validated for } \\
\text { the purpose of } \\
\text { assessing the } \\
\text { presence or } \\
\text { impact of } \\
\text { bladder/bowel } \\
\text { disorders }\end{array}$ \\
\hline
\end{tabular}

This article is protected by copyright. All rights reserved. 


\begin{tabular}{|c|c|c|c|c|}
\hline $\begin{array}{l}\text { Landi } \\
(2016)^{35}\end{array}$ & Interview & $\begin{array}{l}\text { How often do you leak } \\
\text { urine? }\end{array}$ & NA & NA \\
\hline $\begin{array}{l}\text { Leining } \\
(2006)^{36}\end{array}$ & $\begin{array}{l}\text { BCPT } \\
\text { symptom } \\
\text { checklist }\end{array}$ & $\begin{array}{l}\text { How bothered have you } \\
\text { been by difficulty with } \\
\text { bladder control in the } \\
\text { past } 4 \text { weeks. } \\
\text { - SUI: difficulty } \\
\text { with bladder } \\
\text { control at other } \\
\text { times (coughing, } \\
\text { sneezing) }\end{array}$ & Yes & No \\
\hline $\begin{array}{l}\text { Morrow } \\
(2014)^{37}\end{array}$ & $\begin{array}{l}\text { Menopause } \\
\text { symptom } \\
\text { scale from } \\
\text { BCPT } \\
\text { symptom } \\
\text { checklist }\end{array}$ & $\begin{array}{l}\text { How bothered have you } \\
\text { been by difficulty with } \\
\text { bladder control in the } \\
\text { past } 4 \text { weeks. }\end{array}$ & Yes & No \\
\hline $\begin{array}{l}\text { Park } \\
(2013)^{38}\end{array}$ & $\begin{array}{l}\text { Menopause } \\
\text { Rating Scale }\end{array}$ & $\begin{array}{l}\text { Which of the following } \\
\text { apply to you at this } \\
\text { time? Bladder problems } \\
\text { (difficulty in urinating, } \\
\text { increased need to } \\
\text { urinate, bladder } \\
\text { incontinence) }\end{array}$ & Yes & No \\
\hline
\end{tabular}

Appendix 3: Outcome measures used in included studies to assess the presence or impact of bladder and bowel disorders (continued)

\begin{tabular}{|c|c|c|c|c|}
\hline $\begin{array}{c}\text { Author } \\
\text { (year) }\end{array}$ & $\begin{array}{c}\text { Measurement } \\
\text { tool used }\end{array}$ & Question wording & $\begin{array}{c}\text { Validated } \\
\text { questionnaire }\end{array}$ & $\begin{array}{c}\text { Validated for } \\
\text { the purpose of } \\
\text { assessing the } \\
\text { presence or } \\
\text { impact of } \\
\text { bladder/bowel } \\
\text { disorders }\end{array}$ \\
\hline Robinson & QUID & $\begin{array}{l}\text { Do you leak urine } \\
\text { (even small drops), }\end{array}$ & Yes & Yes \\
\hline
\end{tabular}

This article is protected by copyright. All rights reserved. 


\begin{tabular}{|c|c|c|c|c|}
\hline$(2017)^{24}$ & & $\begin{array}{l}\text { wet yourself, or wet } \\
\text { your pads or } \\
\text { undergarments when } \\
\text { you cough or sneeze? }\end{array}$ & & \\
\hline $\begin{array}{l}\text { Schover } \\
(2014)^{39}\end{array}$ & BESS & $\begin{array}{l}\text { How bothered have } \\
\text { you been by difficulty } \\
\text { with bladder control in } \\
\text { the past } 4 \text { weeks. }\end{array}$ & Yes & No \\
\hline $\begin{array}{l}\text { Stanton } \\
(2005)^{40}\end{array}$ & $\begin{array}{l}\text { BCPT } \\
\text { symptom } \\
\text { checklist }\end{array}$ & $\begin{array}{l}\text { How bothered have } \\
\text { you been by difficulty } \\
\text { with bladder control in } \\
\text { the past } 4 \text { weeks. } \\
\text { - SUI: difficulty } \\
\text { with bladder } \\
\text { control at other } \\
\text { times } \\
\text { (coughing, } \\
\text { sneezing) }\end{array}$ & Yes & No \\
\hline
\end{tabular}

SUI, stress urinary incontinence; UUI, urge urinary incontinence; BFLUTS, Bristol Female Lower Urinary Tract Symptoms; BCPT, Breast Cancer Prevention Trial; Breast Cancer Prevention Trial Eight Symptom Scale, BESS; EORTC QLQ-C30, European Organization for Research and Treatment of Cancer quality of life questionnaire; QUID, Questionnaire for Urinary Incontinence Diagnosis; UDI-6, Urinary Distress Inventory-6 questionnaire. CARES, Cancer Rehabilitation Evaluation System; GCS, Greene Climacteric Scale.

Appendix 4: Type of bladder disorder and associated severity of bother

\begin{tabular}{|c|c|c|c|c|c|c|}
\hline \multirow[t]{2}{*}{ Study } & \multirow{2}{*}{$\begin{array}{l}\text { Type of } \\
\text { bladder } \\
\text { disorder }\end{array}$} & \multicolumn{4}{|c|}{$\begin{array}{c}\text { Proportion of women reporting severity of } \\
\text { bother } n,(\%)\end{array}$} & \multirow{2}{*}{$\begin{array}{c}\text { Severity } \\
\text { of bother } \\
\text { score, } \\
\text { mean } \\
\text { (SD) }\end{array}$} \\
\hline & & None & Mild & Moderate & Severe & \\
\hline $\begin{array}{l}\text { Avis } \\
(2004)^{26}\end{array}$ & Stress UI & $150(74)$ & 30 (14) & \multicolumn{2}{|c|}{$24(12)$} & - \\
\hline
\end{tabular}

This article is protected by copyright. All rights reserved. 


\begin{tabular}{|c|c|c|c|c|c|c|}
\hline \multirow{10}{*}{$\begin{array}{l}\text { Baumgart } \\
\text { (2011) }^{25}\end{array}$} & \multirow[t]{2}{*}{ Stress UI } & \multirow[t]{2}{*}{-} & \multirow[t]{2}{*}{-} & \multicolumn{2}{|c|}{ Tam: 24 (73) } & \multirow[t]{2}{*}{-} \\
\hline & & & & \multicolumn{2}{|c|}{ AI: 23 (70) } & \\
\hline & \multirow[t]{2}{*}{ Urge UI } & \multirow[t]{2}{*}{-} & \multirow[t]{2}{*}{-} & \multicolumn{2}{|c|}{ Tam: 19 (56) } & \multirow[t]{2}{*}{-} \\
\hline & & & & \multicolumn{2}{|c|}{ AI: 21 (64) } & \\
\hline & \multirow{2}{*}{$\begin{array}{l}\text { Frequent } \\
\text { urination }\end{array}$} & \multirow[t]{2}{*}{-} & \multirow[t]{2}{*}{-} & \multicolumn{2}{|c|}{ Tam: 23 (68) } & \multirow[t]{2}{*}{-} \\
\hline & & & & AI: & 63) & \\
\hline & \multirow{2}{*}{$\begin{array}{l}\text { Small } \\
\text { amounts of } \\
\text { leakage }\end{array}$} & \multirow[t]{2}{*}{-} & \multirow[t]{2}{*}{-} & \multicolumn{2}{|c|}{ Tam: 24 (47) } & \multirow[t]{2}{*}{-} \\
\hline & & & & \multicolumn{2}{|c|}{ AI: 23 (46) } & \\
\hline & \multirow{2}{*}{$\begin{array}{l}\text { Difficulty } \\
\text { emptying } \\
\text { bladder }\end{array}$} & \multirow[t]{2}{*}{-} & \multirow[t]{2}{*}{-} & \multicolumn{2}{|c|}{ Tam: 6 (18) } & \multirow[t]{2}{*}{-} \\
\hline & & & & \multicolumn{2}{|c|}{ AI: 5 (16) } & \\
\hline $\begin{array}{l}\text { Biglia } \\
(2010)^{18}\end{array}$ & $\begin{array}{l}\text { Urogenital } \\
\text { symptoms }\end{array}$ & - & - & - & - & $3.4(3.7)$ \\
\hline \multirow{3}{*}{$\begin{array}{l}\text { Chin } \\
(2009)^{28}\end{array}$} & Any UI & $158(63)$ & 76 (30) & \multicolumn{2}{|c|}{$15(6)$} & - \\
\hline & $\begin{array}{l}\text { Urinary } \\
\text { urgency }\end{array}$ & $144(57)$ & $68(27)$ & \multicolumn{2}{|c|}{35 (14) } & - \\
\hline & Dysuria & 217 (87) & $26(10)$ & \multicolumn{2}{|c|}{$5(2)$} & - \\
\hline $\begin{array}{l}\text { Couzi } \\
(1995)^{30}\end{array}$ & $\begin{array}{l}\text { Difficulty } \\
\text { with } \\
\text { bladder } \\
\text { control }\end{array}$ & - & $\begin{array}{l}101 \\
(55)\end{array}$ & $40(22)$ & $42(23)$ & - \\
\hline $\begin{array}{l}\text { Daniel } \\
(2014)^{31}\end{array}$ & Bladder & $122(65)$ & $28(15)$ & $26(14)$ & $11(6)$ & - \\
\hline
\end{tabular}

This article is protected by copyright. All rights reserved. 


\begin{tabular}{|c|c|c|c|c|c|c|}
\hline & problems & & & & & \\
\hline \multirow[t]{2}{*}{$\begin{array}{l}\text { Ganz } \\
(2016)\end{array}$} & \multirow[t]{2}{*}{$\begin{array}{l}\text { Bladder } \\
\text { problems }\end{array}$} & \multirow[t]{2}{*}{ - } & \multirow[t]{2}{*}{ - } & \multirow[t]{2}{*}{ - } & \multirow[t]{2}{*}{ - } & Tam: 1 \\
\hline & & & & & & AI: 0.8 \\
\hline $\begin{array}{l}\text { Gupta } \\
\text { (2006) }^{34}\end{array}$ & $\begin{array}{l}\text { Bladder } \\
\text { problems }\end{array}$ & - & - & 78 (39) & - & - \\
\hline
\end{tabular}

Appendix 4: Type of bladder disorder and associated severity of bother (continued)

\begin{tabular}{|c|c|c|c|c|c|c|}
\hline $\begin{array}{l}\text { Kyvernitakis } \\
(2014)^{19}\end{array}$ & $\begin{array}{l}\text { Bladder } \\
\text { problems }\end{array}$ & 107 (67) & 23 (15) & $16(10)$ & $13(8)$ & - \\
\hline \multirow[t]{4}{*}{$\begin{array}{l}\text { Morales } \\
(2004)^{23}\end{array}$} & \multirow[t]{4}{*}{$\begin{array}{l}\text { Urinary } \\
\text { problems }\end{array}$} & $\begin{array}{l}\text { Tam: } 120 \\
\text { (73) }\end{array}$ & - & \multicolumn{2}{|c|}{ Tam: 45 (27) } & - \\
\hline & & $\begin{array}{l}\text { Trial: } 121 \\
\text { (74) }\end{array}$ & & \multicolumn{2}{|c|}{ Trial: 43 (26) } & \\
\hline & & $\begin{array}{l}\text { NSAI: } \\
100(61)\end{array}$ & & \multicolumn{2}{|c|}{ NSAI: 64 (39) } & \\
\hline & & $\begin{array}{c}\text { (N)SAI: } \\
41 \text { (75) }\end{array}$ & & \multicolumn{2}{|c|}{ (N)SAI: 41 (25) } & \\
\hline $\begin{array}{l}\text { Oleske } \\
(2004)^{22}\end{array}$ & $\begin{array}{l}\text { Difficulty } \\
\text { with } \\
\text { bladder } \\
\text { control }\end{array}$ & - & $31(25)$ & $20(16)$ & $5(4)$ & - \\
\hline $\begin{array}{l}\text { Park } \\
(2013)^{38}\end{array}$ & $\begin{array}{l}\text { Bladder } \\
\text { problems }\end{array}$ & - & - & - & - & $3.1(2.4)$ \\
\hline
\end{tabular}

This article is protected by copyright. All rights reserved. 
*Includes both vaginal and urinary symptoms.

UI, urinary incontinence; Tam, tamoxifen; AI, aromatase inhibitor; Trial, receiving tamoxifen or letrozole; NSAI, non-steroidal aromatase inhibitors; (N)SAI, nonsteroidal and steroidal aromatase inhibitors.

Reference list

1. WHO. Breast cancer. 2018 [cited 2020; Available from: https://www.who.int/cancer/prevention/diagnosis-screening/breast-cancer/en/.

2. Ganz, P.A., et al., Physical and psychosocial recovery in the year after primary treatment of breast cancer. Journal of clinical oncology: official journal of the American Society of Clinical Oncology, 2011. 29(9): p. 1101.

3. Lester, J.L. and L.A. Bernhard, Urogenital atrophy in breast cancer survivors. Oncol Nurs Forum, 2009. 36(6): p. 693.

4. Donovan, K.A., et al., Urinary symptoms in breast cancer. CA Cancer J Clin, 2012. 118: p. 582-593.

5. Weber, M.A., et al., Local Oestrogen for Pelvic Floor Disorders: A Systematic Review. PloS one, 2015. 10(9): p. e0136265.

6. Hosmer, D.W. and S. Lemeshow, Applied logistic regression. 3rd ed. ed, ed. R.X. Sturdivant. 2013, Hoboken, N.J.: Hoboken, N.J.: Wiley.

7. Higgins JPT, G.S. Cochrane Handbook for Systematic Reviews of Interventions Version 5.1.0. 2011; Available from: www.handbook.cochrane.org.

8. Moola S, M.Z., Tufanaru C, Aromataris E, Sears K, Sfetcu R, Currie M, Qureshi R, Mattis P, and Lisy K. Chapter 7: Systematic reviews of etiology and risk. Joanna Briggs Institute Reviewer's Manual. 2017; Available from: https://reviewersmanual.joannabriggs.org/.

9. Von Elm, E., et al., The Strengthening the Reporting of Observational Studies in Epidemiology (STROBE) statement: guidelines for reporting observational studies. Lancet (London, England), 2007. 370(9596): p. 1453.

10. Simek, E.M., L. McPhate, and T.P. Haines, Adherence to and efficacy of home exercise programs to prevent falls: A systematic review and meta-analysis of the impact of exercise program characteristics. Preventive Medicine, 2012. 55(4): p. 262-275.

11. Humaidan, P. and N. P. Polyzos, (Meta)analyze this: Systematic reviews might lose credibility. Nature Medicine, 2012. 18(9): p. 1321.

This article is protected by copyright. All rights reserved. 
12. Bown, M.J. and A.J. Sutton, Quality Control in Systematic Reviews and Metaanalyses. Eur J Vasc Endovasc Surg, 2010. 40(5): p. 669-677.

13. Duijts, S.F.A., et al., Efficacy of cognitive behavioral therapy and physical exercise in alleviating treatment-induced menopausal symptoms in patients with breast cancer: results of a randomized, controlled, multicenter trial. Journal of clinical oncology: official journal of the American Society of Clinical Oncology, 2012. 30(33): p. 4124.

14. Ganz, P.A., et al., Managing Menopausal Symptoms in Breast Cancer Survivors: Results of a Randomized Controlled Trial. Journal of the National Cancer Institute, 2000. 92(13): p. 1054-1064.

15. Ganz, P.A., et al., Patient-reported outcomes with anastrozole versus tamoxifen for postmenopausal patients with ductal carcinoma in situ treated with lumpectomy plus radiotherapy (NSABP B-35): a randomised, doubleblind, phase 3 clinical trial. The Lancet, 2016. 387(10021): p. 857-865.

16. Land, S., et al., Health-Related Quality of Life in Axillary Node-Negative, Estrogen Receptor-Negative Breast Cancer Patients Undergoing AC Versus CMF Chemotherapy: Findings from the National Surgical Adjuvant Breast and Bowel Project B-23. Breast Cancer Research and Treatment, 2004. 86(2): p. 153-164.

17. Lötzke, D., et al., Iyengar-Yoga Compared to Exercise as a Therapeutic Intervention during (Neo)adjuvant Therapy in Women with Stage I-III Breast Cancer: Health-Related Quality of Life, Mindfulness, Spirituality, Life Satisfaction, and Cancer-Related Fatigue. Evidence-based complementary and alternative medicine: eCAM, 2016. 2016: p. 5931816.

18. Schover, L.R., et al., Randomized trial of peer counseling on reproductive health in African American breast cancer survivors. Journal of clinical oncology: official journal of the American Society of Clinical Oncology, 2006. 24(10): p. 1620.

19. Versmessen, H., et al., Health-related quality of life in survivors of stage I-II breast cancer: randomized trial of post-operative conventional radiotherapy and hypofractionated tomotherapy. BMC Cancer, 2012. 12(1): p. 495.

20. Alfano, C.M., et al., Psychometric properties of a tool for measuring hormone-related symptoms in breast cancer survivors. Psycho-Oncology, 2006. 15(11): p. 985-1000.

21. Biglia, N., et al., Effects of Surgical and Adjuvant Therapies for Breast Cancer on Sexuality, Cognitive Functions, and Body Weight. The Journal of Sexual Medicine, 2010. 7(5): p. 1891-1900.

22. Kyvernitakis, I., et al., Prevalence of menopausal symptoms and their influence on adherence in women with breast cancer. Climacteric, 2014. 17(3): p. 252-259.

This article is protected by copyright. All rights reserved. 
23. Pagano, T., et al., Fractional microablative CO2 laser in breast cancer survivors affected by iatrogenic vulvovaginal atrophy after failure of nonestrogenic local treatments: a retrospective study. Menopause, 2018. 25(6): p. 657-662.

24. Härtl, K., et al., Personality traits and psychosocial stress: quality of life over 2 years following breast cancer diagnosis and psychological impact factors. Psycho-Oncology, 2010. 19(2): p. 160-169.

25. Oleske, et al., Determination of factors associated with hospitalization in breast cancer survivors. Oncol Nurs Forum, 2004. 31(6): p. 1081-8.

26. Morales, L., et al., Acute effects of tamoxifen and third-generation aromatase inhibitors on menopausal symptoms of breast cancer patients. Anti-Cancer Drugs, 2004. 15(8): p. 753-760.

27. Robinson, P.J., et al., Aromatase Inhibitors Are Associated With Low Sexual Desire Causing Distress and Fecal Incontinence in Women: An Observational Study. J Sex Med, 2017. 14(12): p. 1566-1574.

28. Baumgart, J., et al., Urogenital disorders in women with adjuvant endocrine therapy after early breast cancer. Am J Obstet Gynecol, 2011. 204(1): p. 26.e1-26.e7.

29. Avis, N.E., S. Crawford, and J. Manuel, Psychosocial problems among younger women with breast cancer. Psycho-Oncology, 2004. 13(5): p. 295308.

30. Chen, Q., et al., Health-Related Quality of Life among Women Breast Cancer Patients in Eastern China. BioMed research international, 2018. 2018: p. 1452635.

31. Chin, S.N., et al., Prevalence and Severity of Urogenital Symptoms in Postmenopausal Women Receiving Endocrine Therapy for Breast Cancer. Clinical Breast Cancer, 2009. 9(2): p. 108-117.

32. Cook, D.E., et al., Missing documentation in breast cancer survivors: genitourinary syndrome of menopause. Menopause, 2017. 24(12): p. 13601364.

33. Couzi, R.J., K.J. Helzlsouer, and J.H. Fetting, Prevalence of menopausal symptoms among women with a history of breast cancer and attitudes toward estrogen replacement therapy. J Clin Oncol, 1995. 13(11): p. 2737-44.

34. Daniel, B., et al., Management of Menopausal Symptoms for Breast Cancer Patients. British Journal of Nursing, 2014. 23(8): p. 427-432.

35. Ganz, et al., Breast cancer in younger women: reproductive and late health effects of treatment. J Clin Oncol., 2003. 21(22): p. 4184-93.

36. Greendale, A.G., et al., Factors related to sexual function in postmenopausal women with a history of breast cancer. Menopause, 2001. 8(2): p. 111-119.

This article is protected by copyright. All rights reserved. 
37. Gupta, P., et al., Menopausal symptoms in women treated for breast cancer: the prevalence and severity of symptoms and their perceived effects on quality of life. Climacteric, 2006. 9(1): p. 49-58.

38. Landi, S., et al., Endocrine therapy and urogenital outcomes among women with a breast cancer diagnosis. An International Journal of Studies of Cancer in Human Populations, 2016. 27(11): p. 1325-1332.

39. Leining, M.G., et al., Menopausal-type symptoms in young breast cancer survivors. Annals of Oncology, 2006. 17(12): p. 1777-1782.

40. Morrow, P.K., et al., Effect of Age and Race On Quality of Life in Young Breast Cancer Survivors. Clinical Breast Cancer, 2014. 14(2): p. e21-e31.

41. Park, H. and H. Yoon, Menopausal symptoms, sexual function, depression, and quality of life in Korean patients with breast cancer receiving chemotherapy. Supportive Care in Cancer, 2013. 21(9): p. 2499-2507.

42. Schover, L.R., et al., Sexual Problems During the First 2 Years of Adjuvant Treatment with Aromatase Inhibitors. Journal of Sexual Medicine, 2014. 11(12): p. 3102-3111.

43. Stanton, A.L., C.A. Bernaards, and P.A. Ganz, The BCPT Symptom Scales: A Measure of Physical Symptoms for Women Diagnosed With or at Risk for Breast Cancer. Journal of the National Cancer Institute, 2005. 97(6): p. 448456.

44. Haylen, B., et al., An International Urogynecological Association (IUGA)/International Continence Society (ICS) joint report on the terminology for female pelvic floor dysfunction. Including Pelvic Floor Dysfunction, 2010. 21(1): p. 5-26.

45. Milsom I, et al., Epidemiology of urinary incontinence (UI) and other lowerurinary tract symptoms (LUTS), pelvic organ prolapse (POP)and anal incontinence (AI). 6th ed. 2017 Incontinence: 6th International Consultation on Incontinence, Tokyo, September 2016: p. 18-22.

46. Van Oyen, H. and P. Van Oyen, Urinary incontinence in Belgium; prevalence, correlates and psychosocial consequences. Acta Clinica Belgica, 2002. 57(4): p. 207-218.

47. Ditah, I., et al., Prevalence, Trends, and Risk Factors for Fecal Incontinence in United States Adults, 2005-2010. Clinical Gastroenterology and Hepatology, 2014. 12(4): p. 636-643.e2.

48. Minassian, A.V., et al., Severity of Urinary Incontinence and Effect on Quality of Life in Women by Incontinence Type. Obstetrics \& Gynecology, 2013. 121(5): p. 1083-1090.

49. Neron, M., et al., Impact of gynecologic cancer on pelvic floor disorder symptoms and quality of life: an observational study. Scientific reports, 2019. 9(1): p. 2250.

This article is protected by copyright. All rights reserved. 
50. Knobf, M.T., The Transition Experience to Breast Cancer Survivorship. Seminars in Oncology Nursing, 2015. 31(2): p. 178-182.

Table 1: Risk of bias assessment

\begin{tabular}{|c|c|c|c|c|c|c|c|c|c|c|c|c|c|}
\hline \multirow{2}{*}{$\begin{array}{l}\text { Aut } \\
\text { hor } \\
\text { (ye } \\
\text { ar) }\end{array}$} & \multicolumn{5}{|c|}{ Sample characteristics } & \multicolumn{2}{|c|}{$\begin{array}{c}\text { Confound } \\
\text { ers }\end{array}$} & \multicolumn{6}{|c|}{ Outcomes and analysis } \\
\hline & 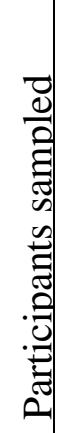 & 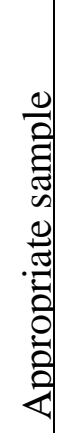 & 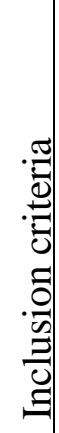 & 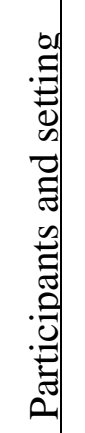 & 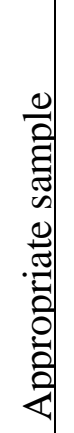 & 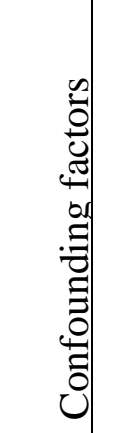 & 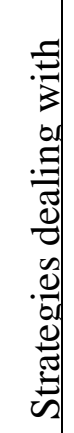 & 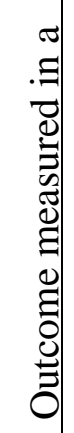 & 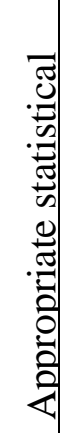 & 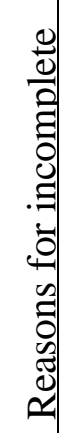 & 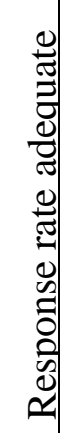 & 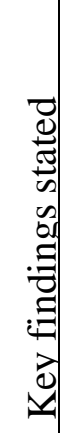 & 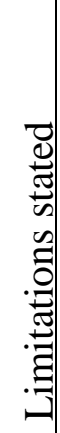 \\
\hline
\end{tabular}

Randomised controlled studies

\begin{tabular}{|c|c|c|c|c|c|c|c|c|c|c|c|c|c|}
\hline $\begin{array}{l}\text { Duijts } \\
(2012 \\
)^{10}\end{array}$ & $\mathrm{Y}$ & $\mathrm{Y}$ & $\mathrm{Y}$ & $\mathrm{Y}$ & $\mathrm{Y}$ & $\mathrm{Y}$ & $\mathrm{Y}$ & $\mathrm{Y}$ & $\mathrm{U}$ & $\mathrm{N}$ & $\mathrm{Y}$ & $Y$ & $\mathrm{Y}$ \\
\hline $\begin{array}{l}\text { Ganz } \\
(2000 \\
)^{11}\end{array}$ & $\mathrm{Y}$ & $\mathrm{Y}$ & $\mathrm{Y}$ & $\mathrm{Y}$ & $\mathrm{Y}$ & $\mathrm{Y}$ & $\mathrm{Y}$ & $\mathrm{N}$ & $\mathrm{U}$ & $\mathrm{Y}$ & $\mathrm{Y}$ & $\mathrm{Y}$ & $\mathrm{Y}$ \\
\hline $\begin{array}{l}\text { Ganz } \\
(2016 \\
)^{12}\end{array}$ & $\mathrm{Y}$ & $\mathrm{Y}$ & $\mathrm{Y}$ & $\mathrm{Y}$ & $\mathrm{Y}$ & $\mathrm{Y}$ & $\mathrm{Y}$ & $\mathrm{N}$ & $\mathrm{U}$ & $\mathrm{N}$ & $\mathrm{Y}$ & $Y$ & $\mathrm{Y}$ \\
\hline $\begin{array}{l}\text { Land } \\
(2004 \\
)^{13}\end{array}$ & $\mathrm{Y}$ & $\mathrm{U}$ & $\mathrm{Y}$ & $\mathrm{Y}$ & $\mathrm{Y}$ & $\mathrm{Y}$ & $\mathrm{Y}$ & $\mathrm{N}$ & $\mathrm{U}$ & $\mathrm{N}$ & $\mathrm{Y}$ & $\mathrm{Y}$ & $\mathrm{Y}$ \\
\hline $\begin{array}{l}\text { Lötzk } \\
\text { e } \\
(2016 \\
)^{14}\end{array}$ & $\mathrm{Y}$ & $\mathrm{Y}$ & $\mathrm{Y}$ & $\mathrm{Y}$ & $\mathrm{Y}$ & $\mathrm{N}$ & $\mathrm{N}$ & $\mathrm{N}$ & $\mathrm{U}$ & $\mathrm{N}$ & $\mathrm{N}$ & $Y$ & $\mathrm{Y}$ \\
\hline
\end{tabular}

This article is protected by copyright. All rights reserved. 


\begin{tabular}{|l|c|c|c|c|c|c|c|c|c|c|c|c|c|}
\hline $\begin{array}{l}\text { Schov } \\
\text { er } \\
)^{15}\end{array}$ & $\mathrm{Y}$ & $\mathrm{Y}$ & $\mathrm{Y}$ & $\mathrm{Y}$ & $\mathrm{N}$ & $\mathrm{Y}$ & $\mathrm{Y}$ & $\mathrm{N}$ & $\mathrm{Y}$ & $\mathrm{N}$ & $\mathrm{Y}$ & $\mathrm{Y}$ & $\mathrm{Y}$ \\
\hline $\begin{array}{l}\text { Vers } \\
\text { messe } \\
\text { (2012 } \\
)^{16}\end{array}$ & $\mathrm{Y}$ & $\mathrm{Y}$ & $\mathrm{Y}$ & $\mathrm{Y}$ & $\mathrm{N}$ & $\mathrm{N}$ & $\mathrm{N}$ & $\mathrm{N}$ & $\mathrm{Y}$ & $\mathrm{Y}$ & $\mathrm{Y}$ & $\mathrm{Y}$ & $\mathrm{Y}$ \\
\hline
\end{tabular}

\section{Cohort studies}

\begin{tabular}{|c|c|c|c|c|c|c|c|c|c|c|c|c|c|}
\hline $\begin{array}{l}\text { Alfan } \\
\text { o } \\
(2006 \\
)^{17}\end{array}$ & $\mathrm{Y}$ & $\mathrm{Y}$ & $\mathrm{Y}$ & $\mathrm{Y}$ & $\mathrm{Y}$ & $\mathrm{Y}$ & $\mathrm{Y}$ & $\mathrm{N}$ & $\mathrm{U}$ & $\mathrm{Y}$ & Y & $\mathrm{Y}$ & $\mathrm{Y}$ \\
\hline $\begin{array}{l}\text { Biglia } \\
(2010 \\
)^{18}\end{array}$ & $\mathrm{~N}$ & $\mathrm{Y}$ & $\mathrm{Y}$ & $\mathrm{Y}$ & $\mathrm{N}$ & $\mathrm{Y}$ & $\mathrm{Y}$ & $\mathrm{N}$ & $\mathrm{U}$ & $\mathrm{N}$ & NA & $\mathrm{Y}$ & $\mathrm{Y}$ \\
\hline $\begin{array}{l}\text { Hartl } \\
(2010 \\
)^{21}\end{array}$ & $\mathrm{Y}$ & $\mathrm{Y}$ & $\mathrm{Y}$ & $\mathrm{Y}$ & $\mathrm{N}$ & $\mathrm{N}$ & $\mathrm{N}$ & $\mathrm{N}$ & $\mathrm{Y}$ & $\mathrm{N}$ & $\mathrm{Y}$ & $\mathrm{Y}$ & $\mathrm{Y}$ \\
\hline $\begin{array}{l}\text { Kyver } \\
\text { nitaki } \\
\text { s } \\
(2014 \\
)^{19}\end{array}$ & $\mathrm{U}$ & $\mathrm{U}$ & $\mathrm{N}$ & $\mathrm{N}$ & $\mathrm{N}$ & $\mathrm{N}$ & $\mathrm{N}$ & $\mathrm{N}$ & $\mathrm{Y}$ & $\mathrm{N}$ & $\mathrm{Y}$ & $\mathrm{Y}$ & $\mathrm{Y}$ \\
\hline $\begin{array}{l}\text { Ganz } \\
(2011 \\
)^{2}\end{array}$ & $\mathrm{U}$ & $\mathrm{Y}$ & $\mathrm{N}$ & $\mathrm{N}$ & $\mathrm{Y}$ & $\mathrm{Y}$ & $\mathrm{Y}$ & $\mathrm{N}$ & $\mathrm{Y}$ & $\mathrm{N}$ & $\mathrm{Y}$ & $\mathrm{Y}$ & $\mathrm{Y}$ \\
\hline $\begin{array}{l}\text { Moral } \\
\text { es } \\
(2004 \\
)^{23}\end{array}$ & $\mathrm{Y}$ & $Y$ & $\mathrm{~N}$ & $\mathrm{Y}$ & $\mathrm{N}$ & $\mathrm{N}$ & $\mathrm{N}$ & $\mathrm{N}$ & $Y$ & $\mathrm{Y}$ & $\mathrm{Y}$ & $\mathrm{Y}$ & $\mathrm{Y}$ \\
\hline
\end{tabular}

This article is protected by copyright. All rights reserved. 


\begin{tabular}{|l|c|c|c|c|c|c|c|c|c|c|c|c|c|}
\hline $\begin{array}{l}\text { Olesk } \\
(2004 \\
)^{22}\end{array}$ & $\mathrm{Y}$ & $\mathrm{U}$ & $\mathrm{Y}$ & $\mathrm{N}$ & $\mathrm{N}$ & $\mathrm{Y}$ & $\mathrm{Y}$ & $\mathrm{N}$ & $\mathrm{U}$ & $\mathrm{Y}$ & $\mathrm{Y}$ & $\mathrm{Y}$ & $\mathrm{Y}$ \\
\hline $\begin{array}{l}\text { Pagan } \\
\text { (2018 } \\
)^{20}\end{array}$ & $\mathrm{Y}$ & $\mathrm{U}$ & $\mathrm{U}$ & $\mathrm{Y}$ & $\mathrm{N}$ & $\mathrm{Y}$ & $\mathrm{Y}$ & $\mathrm{N}$ & $\mathrm{N}$ & $\mathrm{Y}$ & $\mathrm{Y}$ & $\mathrm{Y}$ & $\mathrm{Y}$ \\
\hline
\end{tabular}

Y, yes; N, no; U, unclear; NA, not applicable.

Table 1: Risk of bias assessment (continued)

\begin{tabular}{|c|c|c|c|c|c|c|c|c|c|c|c|c|c|}
\hline \multirow{2}{*}{$\begin{array}{l}\text { A } \\
\text { ut } \\
\text { ho } \\
\text { r } \\
\text { (y } \\
\text { ea } \\
\text { r) }\end{array}$} & \multicolumn{5}{|c|}{ Sample characteristics } & \multicolumn{2}{|c|}{$\begin{array}{c}\text { Confound } \\
\text { ers }\end{array}$} & \multicolumn{6}{|c|}{ Outcomes and analysis } \\
\hline & 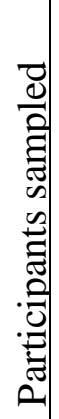 & 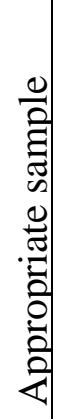 & 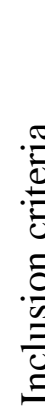 & 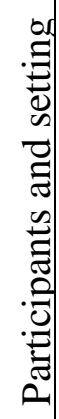 & 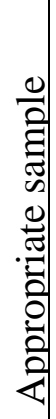 & 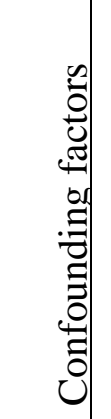 & 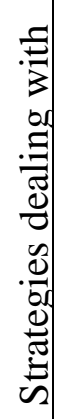 & 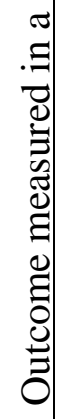 & 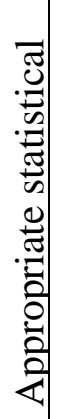 & $\begin{array}{c}0 \\
0 \\
0 \\
0 \\
0 \\
0 \\
.0 \\
0 \\
0 \\
0 \\
0 \\
0 \\
0 \\
0 \\
0\end{array}$ & 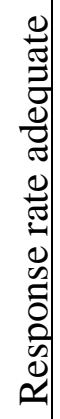 & 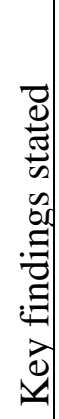 & 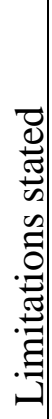 \\
\hline
\end{tabular}

\section{Cross-sectional studies}

\begin{tabular}{|l|c|c|c|c|c|c|c|c|c|c|c|c|c|}
\hline $\begin{array}{l}\text { Avis } \\
(200 \\
4)^{26}\end{array}$ & $\mathrm{Y}$ & $\mathrm{Y}$ & $\mathrm{Y}$ & $\mathrm{Y}$ & $\mathrm{N}$ & $\mathrm{NA}$ & $\mathrm{NA}$ & $\mathrm{N}$ & $\mathrm{Y}$ & $\mathrm{N}$ & $\mathrm{Y}$ & $\mathrm{Y}$ & $\mathrm{Y}$ \\
\hline $\begin{array}{l}\text { Bau } \\
\text { mga } \\
\mathrm{rt} \\
(201 \\
1)^{25}\end{array}$ & $\mathrm{Y}$ & $\mathrm{Y}$ & $\mathrm{Y}$ & $\mathrm{Y}$ & $\mathrm{N}$ & $\mathrm{Y}$ & $\mathrm{Y}$ & $\mathrm{Y}$ & $\mathrm{Y}$ & $\mathrm{N}$ & $\mathrm{N}$ & $\mathrm{Y}$ & $\mathrm{Y}$ \\
\hline $\begin{array}{l}\text { Che } \\
\mathrm{n}\end{array}$ & $\mathrm{Y}$ & $\mathrm{Y}$ & $\mathrm{Y}$ & $\mathrm{Y}$ & $\mathrm{Y}$ & $\mathrm{NA}$ & $\mathrm{NA}$ & $\mathrm{N}$ & $\mathrm{Y}$ & $\mathrm{N}$ & $\mathrm{U}$ & $\mathrm{Y}$ & $\mathrm{Y}$ \\
$\begin{array}{l}(201 \\
8)^{27}\end{array}$ & & & & & & & & & & & \\
\hline
\end{tabular}

This article is protected by copyright. All rights reserved. 


\begin{tabular}{|c|c|c|c|c|c|c|c|c|c|c|c|c|c|}
\hline $\begin{array}{l}\text { Chin } \\
(200 \\
9)^{28}\end{array}$ & $\mathrm{Y}$ & $\mathrm{Y}$ & $\mathrm{Y}$ & $\mathrm{Y}$ & $\mathrm{Y}$ & NA & NA & $\mathrm{N}$ & $\mathrm{U}$ & $\mathrm{N}$ & $\mathrm{N}$ & $\mathrm{Y}$ & $\mathrm{Y}$ \\
\hline $\begin{array}{l}\text { Coo } \\
\text { k } \\
(201 \\
7)^{29}\end{array}$ & NA & $\mathrm{Y}$ & $\mathrm{Y}$ & $\mathrm{Y}$ & $\mathrm{Y}$ & NA & NA & $\mathrm{N}$ & $\mathrm{Y}$ & NA & NA & $\mathrm{Y}$ & $\mathrm{Y}$ \\
\hline $\begin{array}{l}\text { Cou } \\
\text { zi } \\
(199 \\
5)^{30}\end{array}$ & $\mathrm{Y}$ & $\mathrm{Y}$ & $\mathrm{Y}$ & $\mathrm{U}$ & $\mathrm{Y}$ & NA & NA & $\mathrm{N}$ & $\mathrm{Y}$ & $\mathrm{Y}$ & $\mathrm{Y}$ & $\mathrm{Y}$ & $\mathrm{N}$ \\
\hline $\begin{array}{l}\text { Dani } \\
\text { el } \\
(201 \\
4)^{31}\end{array}$ & $\mathrm{Y}$ & $\mathrm{Y}$ & $\mathrm{Y}$ & $\mathrm{Y}$ & $\mathrm{N}$ & NA & NA & $\mathrm{N}$ & $\mathrm{U}$ & $\mathrm{N}$ & $\mathrm{Y}$ & $\mathrm{Y}$ & $\mathrm{N}$ \\
\hline $\begin{array}{l}\text { Gan } \\
\text { z } \\
(200 \\
3)^{32}\end{array}$ & $\mathrm{Y}$ & $\mathrm{Y}$ & $\mathrm{Y}$ & $\mathrm{N}$ & $Y$ & NA & NA & $\mathrm{N}$ & $\mathrm{U}$ & $\mathrm{Y}$ & $\mathrm{Y}$ & $\mathrm{Y}$ & $\mathrm{Y}$ \\
\hline $\begin{array}{l}\text { Gree } \\
\text { ndal } \\
\text { e } \\
(200 \\
1)^{33}\end{array}$ & $\mathrm{Y}$ & $\mathrm{Y}$ & $\mathrm{Y}$ & $\mathrm{Y}$ & $\mathrm{N}$ & NA & NA & $\mathrm{N}$ & $\mathrm{U}$ & $\mathrm{N}$ & $\mathrm{N}$ & $\mathrm{Y}$ & $\mathrm{Y}$ \\
\hline $\begin{array}{l}\text { Gupt } \\
\text { a } \\
(200 \\
6)^{34}\end{array}$ & $\mathrm{U}$ & $\mathrm{Y}$ & $\mathrm{U}$ & $\mathrm{Y}$ & $\mathrm{N}$ & NA & NA & $\mathrm{N}$ & $\mathrm{U}$ & $\mathrm{N}$ & $\mathrm{Y}$ & $\mathrm{Y}$ & $\mathrm{N}$ \\
\hline $\begin{array}{l}\text { Lan } \\
\text { di } \\
(201 \\
6)^{35}\end{array}$ & $\mathrm{Y}$ & $\mathrm{Y}$ & $\mathrm{Y}$ & $\mathrm{Y}$ & $\mathrm{Y}$ & NA & NA & $\mathrm{N}$ & $\mathrm{Y}$ & $\mathrm{Y}$ & $\mathrm{Y}$ & $\mathrm{Y}$ & $\mathrm{Y}$ \\
\hline
\end{tabular}

This article is protected by copyright. All rights reserved. 


\begin{tabular}{|l|c|c|c|c|c|c|c|c|c|c|c|c|c|}
\hline $\begin{array}{l}\text { Lein } \\
\text { ing } \\
(200 \\
6)^{36}\end{array}$ & $\mathrm{~N}$ & $\mathrm{Y}$ & $\mathrm{Y}$ & $\mathrm{U}$ & $\mathrm{Y}$ & $\mathrm{NA}$ & $\mathrm{NA}$ & $\mathrm{N}$ & $\mathrm{U}$ & $\mathrm{Y}$ & $\mathrm{N}$ & $\mathrm{Y}$ & $\mathrm{Y}$ \\
\hline $\begin{array}{l}\text { Mor } \\
\text { row } \\
\left(\begin{array}{l}201 \\
4)^{37}\end{array}\right.\end{array}$ & $\mathrm{Y}$ & $\mathrm{Y}$ & $\mathrm{Y}$ & $\mathrm{Y}$ & $\mathrm{Y}$ & $\mathrm{NA}$ & $\mathrm{NA}$ & $\mathrm{N}$ & $\mathrm{Y}$ & $\mathrm{Y}$ & $\mathrm{N}$ & $\mathrm{Y}$ & $\mathrm{Y}$ \\
\hline $\begin{array}{l}\text { Park } \\
(201 \\
3)^{38}\end{array}$ & $\mathrm{~N}$ & $\mathrm{Y}$ & $\mathrm{Y}$ & $\mathrm{Y}$ & $\mathrm{N}$ & $\mathrm{NA}$ & $\mathrm{NA}$ & $\mathrm{N}$ & $\mathrm{Y}$ & $\mathrm{N}$ & $\mathrm{U}$ & $\mathrm{Y}$ & $\mathrm{N}$ \\
\hline $\begin{array}{l}\text { Robi } \\
\text { nson } \\
(201 \\
7)^{24}\end{array}$ & $\mathrm{Y}$ & $\mathrm{U}$ & $\mathrm{U}$ & $\mathrm{U}$ & $\mathrm{Y}$ & $\mathrm{NA}$ & $\mathrm{NA}$ & $\mathrm{Y}$ & $\mathrm{Y}$ & $\mathrm{Y}$ & $\mathrm{Y}$ & $\mathrm{Y}$ & $\mathrm{Y}$ \\
\hline $\begin{array}{l}\text { Scho } \\
\text { ver } \\
(201 \\
4)^{39}\end{array}$ & $\mathrm{Y}$ & $\mathrm{Y}$ & $\mathrm{Y}$ & $\mathrm{N}$ & $\mathrm{N}$ & $\mathrm{NA}$ & $\mathrm{NA}$ & $\mathrm{N}$ & $\mathrm{Y}$ & $\mathrm{Y}$ & $\mathrm{N}$ & $\mathrm{Y}$ & $\mathrm{Y}$ \\
\hline $\begin{array}{l}\text { Stan } \\
\text { ton }\end{array}$ & $\mathrm{N}$ & $\mathrm{Y}$ & $\mathrm{Y}$ & $\mathrm{N}$ & $\mathrm{Y}$ & $\mathrm{NA}$ & $\mathrm{NA}$ & $\mathrm{N}$ & $\mathrm{U}$ & $\mathrm{N}$ & $\mathrm{U}$ & $\mathrm{U}$ & $\mathrm{U}$ \\
\hline
\end{tabular}

Y, yes; N, no; U, unclear; NA, not applicable.

Table 2: Characteristics of included studies

\begin{tabular}{|c|c|c|c|c|c|c|c|c|c|c|}
\hline \multicolumn{2}{|c|}{ Study } & \multicolumn{3}{|c|}{ Population } & \multicolumn{3}{c|}{ Exposure } & \multicolumn{3}{c|}{ Outcomes } \\
\hline Aut & Co & Sa & M & Menopa & S & Treatment type & To & As & & \\
hor & unt & m & ea & usal & t & & ol & se & & \\
(yea & ry & pl & n & status & a & & & ss & & \\
r) & & e & ag & (\% of & g & & & m & & \\
\hline
\end{tabular}

This article is protected by copyright. All rights reserved. 


\begin{tabular}{|c|c|c|c|c|c|c|c|c|c|c|c|c|c|c|c|c|}
\hline & & siz & e & & $\mathrm{ome}$ & & & & & & & & en & & & \\
\hline & & & & $\begin{array}{l}\mathbf{P} \\
\mathbf{r} \\
\mathbf{e}\end{array}$ & $\begin{array}{l}\mathbf{P} \\
\mathbf{e} \\
\mathbf{r} \\
\mathbf{i}\end{array}$ & $\begin{array}{l}\mathbf{P} \\
\mathbf{0} \\
\mathrm{S} \\
\mathbf{t}\end{array}$ & $\begin{array}{l}\mathbf{e} \\
\mathbf{a} \\
\mathbf{s} \\
\mathbf{t} \\
\mathbf{c} \\
\mathbf{a} \\
\mathbf{n} \\
\mathbf{c} \\
\mathbf{e} \\
\mathbf{r}\end{array}$ & $\begin{array}{c}\text { Su } \\
\text { rg } \\
\text { er } \\
y\end{array}$ & $\begin{array}{c}\text { C } \\
\text { he } \\
\text { m } \\
\text { o }\end{array}$ & $\begin{array}{c}\mathbf{R} \\
\mathbf{a} \\
\mathbf{d i} \\
\mathbf{0}\end{array}$ & $\begin{array}{l}\text { E } \\
\text { n } \\
\text { d } \\
\text { o }\end{array}$ & & int & & & \\
\hline & & & & & & and & mis & d cor & trol & ed $t$ & als & & & & & \\
\hline $\begin{array}{l}\text { Dui } \\
\text { jts } \\
(201 \\
2)^{10}\end{array}$ & $\begin{array}{c}\text { Net } \\
\text { herl } \\
\text { and } \\
\text { s }\end{array}$ & $\begin{array}{c}42 \\
2\end{array}$ & $\begin{array}{c}48 \\
.2\end{array}$ & $\begin{array}{l}1 \\
0 \\
0\end{array}$ & - & - & $\begin{array}{l}\text { I- } \\
\text { IV }\end{array}$ & $\begin{array}{c}\mathrm{Ye} \\
\mathrm{s}\end{array}$ & $\begin{array}{l}\mathrm{Y} \\
\text { es }\end{array}$ & $\begin{array}{l}\mathrm{N} \\
\mathrm{O}\end{array}$ & $\begin{array}{l}\mathrm{Y} \\
\mathrm{e} \\
\mathrm{s}\end{array}$ & $\begin{array}{l}\text { BF } \\
\text { LU } \\
\text { TS }\end{array}$ & Post & $x$ & $x$ & $\checkmark$ \\
\hline $\begin{array}{l}\text { Gan } \\
\text { Z } \\
(200 \\
0)^{11}\end{array}$ & $\begin{array}{c}\text { US } \\
\text { A }\end{array}$ & 72 & $\begin{array}{c}54 \\
.5\end{array}$ & $\begin{array}{l}\mathrm{N} \\
\mathrm{O}\end{array}$ & $\begin{array}{l}\text { Y } \\
\text { e } \\
\text { s }\end{array}$ & $\begin{array}{l}\mathrm{Y} \\
\mathrm{e} \\
\mathrm{s}\end{array}$ & $\begin{array}{c}\text { I } \\
\text { or } \\
\text { II }\end{array}$ & $\begin{array}{c}Y e \\
\mathrm{~s}\end{array}$ & $\begin{array}{l}\mathrm{Y} \\
\text { es }\end{array}$ & $\begin{array}{l}\mathrm{Y} \\
\text { es }\end{array}$ & $\begin{array}{l}\mathrm{Y} \\
\mathrm{e} \\
\mathrm{s}\end{array}$ & $\begin{array}{l}\text { M } \\
\text { SS }\end{array}$ & Post & $\checkmark$ & $x$ & $\checkmark$ \\
\hline $\begin{array}{l}\text { Gan } \\
z \\
(201 \\
6)^{12}\end{array}$ & $\begin{array}{c}\text { US } \\
\text { A }\end{array}$ & $\begin{array}{c}1 \\
19 \\
3\end{array}$ & $\begin{array}{c}\mathrm{N} \\
\mathrm{R}^{\alpha}\end{array}$ & - & - & $\begin{array}{l}1 \\
0 \\
0\end{array}$ & $\begin{array}{c}\mathrm{D} \\
\mathrm{CI} \\
\mathrm{S}\end{array}$ & $\begin{array}{c}\mathrm{Ye} \\
\mathrm{s}\end{array}$ & $\begin{array}{c}\mathrm{N} \\
\mathrm{O}\end{array}$ & $\begin{array}{l}\mathrm{Y} \\
\mathrm{es}\end{array}$ & $\begin{array}{l}\mathrm{N} \\
\mathrm{O}\end{array}$ & $\begin{array}{l}\text { BC } \\
\text { PT }\end{array}$ & Post & $x$ & $x$ & $\checkmark$ \\
\hline $\begin{array}{l}\text { Lan } \\
\text { d } \\
(200 \\
4)^{13}\end{array}$ & $\begin{array}{c}\text { US } \\
\text { A }\end{array}$ & $\begin{array}{c}16 \\
0\end{array}$ & $\begin{array}{l}\mathrm{N} \\
\mathrm{R}^{\beta}\end{array}$ & $\begin{array}{l}\mathrm{N} \\
\mathrm{R}\end{array}$ & $\begin{array}{l}\mathrm{N} \\
\mathrm{R}\end{array}$ & $\begin{array}{l}\mathrm{N} \\
\mathrm{R}\end{array}$ & $\begin{array}{l}\mathrm{N} \\
\mathrm{R}\end{array}$ & $\begin{array}{c}\mathrm{Ye} \\
\mathrm{s}\end{array}$ & $\begin{array}{l}\mathrm{N} \\
\mathrm{O}\end{array}$ & $\begin{array}{l}\mathrm{N} \\
\mathrm{O}\end{array}$ & $\begin{array}{l}\mathrm{N} \\
\mathrm{O}\end{array}$ & $\begin{array}{l}\text { BC } \\
\text { PT }\end{array}$ & NR & $\checkmark$ & $x$ & $x$ \\
\hline $\begin{array}{l}\text { Löt } \\
\text { zke } \\
(201 \\
6)^{14}\end{array}$ & $\begin{array}{c}\text { Ger } \\
\text { man } \\
\mathrm{y}\end{array}$ & 92 & $\begin{array}{c}51 \\
.0\end{array}$ & $\begin{array}{l}N \\
R\end{array}$ & $\begin{array}{l}\mathrm{N} \\
\mathrm{R}\end{array}$ & $\begin{array}{l}\mathrm{N} \\
\mathrm{R}\end{array}$ & $\begin{array}{l}\text { I- } \\
\text { III }\end{array}$ & No & $\begin{array}{l}\mathrm{Y} \\
\text { es }\end{array}$ & $\begin{array}{l}\mathrm{Y} \\
\text { es }\end{array}$ & $\begin{array}{l}\mathrm{Y} \\
\mathrm{e} \\
\mathrm{s}\end{array}$ & $\begin{array}{c}\text { EO } \\
\text { RT } \\
\text { C } \\
\text { QL } \\
\text { Q- } \\
\text { C3 }\end{array}$ & Peri & $x$ & $x$ & $\checkmark$ \\
\hline
\end{tabular}

This article is protected by copyright. All rights reserved. 


\begin{tabular}{|c|c|c|c|c|c|c|c|c|c|c|c|c|c|c|c|c|}
\hline & & & & & & & & & & & & 0 & & & & \\
\hline $\begin{array}{l}\text { Sch } \\
\text { ove } \\
\text { r } \\
(200 \\
6)^{15}\end{array}$ & $\begin{array}{c}\text { US } \\
\text { A }\end{array}$ & 48 & $\begin{array}{c}49 \\
.3\end{array}$ & $\begin{array}{l}1 \\
9\end{array}$ & $\begin{array}{l}\mathrm{N} \\
\mathrm{R}\end{array}$ & $\begin{array}{l}\mathrm{N} \\
\mathrm{R}\end{array}$ & $\begin{array}{l}0- \\
\text { III }\end{array}$ & $\begin{array}{c}\mathrm{Ye} \\
\mathrm{s}\end{array}$ & $\begin{array}{c}\mathrm{Y} \\
\text { es }\end{array}$ & $\begin{array}{c}\mathrm{N} \\
\mathrm{O}\end{array}$ & $\begin{array}{l}\text { Y } \\
\text { e } \\
\text { s }\end{array}$ & $\begin{array}{l}\mathrm{M} \\
\mathrm{SS}\end{array}$ & Peri & $x$ & $x$ & $\checkmark$ \\
\hline $\begin{array}{l}\text { Ver } \\
\text { sme } \\
\text { ssen } \\
(201 \\
2)^{16}\end{array}$ & $\begin{array}{c}\text { Bel } \\
\text { giu } \\
\text { m }\end{array}$ & $\begin{array}{c}12 \\
1\end{array}$ & $\begin{array}{c}55 \\
.0\end{array}$ & $\begin{array}{l}\mathrm{N} \\
\mathrm{R}\end{array}$ & $\begin{array}{l}\mathrm{N} \\
\mathrm{R}\end{array}$ & $\begin{array}{l}\mathrm{N} \\
\mathrm{R}\end{array}$ & $\begin{array}{l}\text { I- } \\
\text { II }\end{array}$ & $\begin{array}{c}\mathrm{Ye} \\
\mathrm{s}\end{array}$ & $\begin{array}{l}\mathrm{N} \\
\mathrm{O}\end{array}$ & $\begin{array}{l}\mathrm{Y} \\
\text { es }\end{array}$ & $\begin{array}{l}\mathrm{Y} \\
\mathrm{e} \\
\mathrm{s}\end{array}$ & $\begin{array}{c}\text { EO } \\
\text { RT } \\
\text { C } \\
\text { QL } \\
\text { Q- } \\
\text { C3 } \\
0\end{array}$ & Post & $x$ & $x$ & $\checkmark$ \\
\hline \multicolumn{17}{|c|}{ Cohort studies } \\
\hline $\begin{array}{l}\text { Alfa } \\
\text { no } \\
(200 \\
6)^{17}\end{array}$ & $\begin{array}{c}\text { US } \\
\text { A }\end{array}$ & $\begin{array}{c}80 \\
3\end{array}$ & $\begin{array}{c}56 \\
.0\end{array}$ & $\begin{array}{l}1 \\
8\end{array}$ & $\begin{array}{l}\mathrm{N} \\
\mathrm{R}\end{array}$ & $\begin{array}{l}7 \\
5\end{array}$ & $\begin{array}{l}\mathrm{N} \\
\mathrm{R}\end{array}$ & $\begin{array}{c}\mathrm{Ye} \\
\mathrm{s}\end{array}$ & $\begin{array}{c}\mathrm{Y} \\
\text { es }\end{array}$ & $\begin{array}{l}\mathrm{Y} \\
\text { es }\end{array}$ & $\begin{array}{l}\mathrm{N} \\
\mathrm{O}\end{array}$ & $\begin{array}{l}\mathrm{BC} \\
\mathrm{PT}\end{array}$ & Post & $\checkmark$ & $x$ & $\checkmark$ \\
\hline
\end{tabular}

Table 2: Characteristics of included studies (continued)

\begin{tabular}{|c|c|c|c|c|c|c|c|c|c|c|c|c|c|c|c|c|}
\hline $\begin{array}{l}\text { Biglia } \\
(2010)^{18}\end{array}$ & Italy & $\begin{array}{l}3 \\
5\end{array}$ & $\begin{array}{l}45 \\
.7\end{array}$ & $\begin{array}{l}9 \\
1\end{array}$ & - & 9 & NR & $\begin{array}{l}\mathrm{Y} \\
\text { es }\end{array}$ & $\begin{array}{l}\mathrm{Y} \\
\text { es }\end{array}$ & $\begin{array}{l}\mathrm{Y} \\
\text { es }\end{array}$ & $\begin{array}{l}\mathrm{Y} \\
\mathrm{es}\end{array}$ & GCS & $\begin{array}{l}\text { Po } \\
\text { st }\end{array}$ & $x$ & $x$ & $\checkmark$ \\
\hline $\begin{array}{l}\text { Hartl } \\
(2010)^{21}\end{array}$ & $\begin{array}{l}\text { Germ } \\
\text { any }\end{array}$ & $\begin{array}{l}2 \\
0 \\
3\end{array}$ & $\begin{array}{l}58 \\
.4\end{array}$ & $\begin{array}{l}2 \\
2\end{array}$ & - & $\begin{array}{l}7 \\
8\end{array}$ & $\begin{array}{l}\text { I- } \\
\text { IV, } \\
\text { DC } \\
\text { IS }\end{array}$ & $\begin{array}{l}\mathrm{Y} \\
\text { es }\end{array}$ & $\begin{array}{l}\text { Y } \\
\text { es }\end{array}$ & $\begin{array}{l}\text { Y } \\
\text { es }\end{array}$ & $\begin{array}{l}\mathrm{Y} \\
\text { es }\end{array}$ & $\begin{array}{c}\text { EOR } \\
\text { TC } \\
\text { QL } \\
\text { Q- } \\
\text { C30 }\end{array}$ & $\begin{array}{l}\text { Po } \\
\text { st }\end{array}$ & $x$ & $x$ & $\checkmark$ \\
\hline $\begin{array}{l}\text { Kyvern } \\
\text { itakis } \\
(2014)^{19}\end{array}$ & $\begin{array}{l}\text { Germ } \\
\text { any }\end{array}$ & $\begin{array}{l}1 \\
8 \\
0\end{array}$ & $\begin{array}{c}63 \\
.2\end{array}$ & - & - & $\begin{array}{l}1 \\
0 \\
0\end{array}$ & $\begin{array}{l}\text { I- } \\
\text { IV }\end{array}$ & $\begin{array}{l}\mathrm{Y} \\
\text { es }\end{array}$ & $\begin{array}{l}\text { Y } \\
\text { es }\end{array}$ & $\begin{array}{l}\mathrm{Y} \\
\text { es }\end{array}$ & $\begin{array}{c}\mathrm{N} \\
\mathrm{O}\end{array}$ & $\begin{array}{c}\mathrm{MR} \\
\mathrm{S}\end{array}$ & $\begin{array}{l}\mathrm{Pe} \\
\text { ri }\end{array}$ & $\checkmark$ & $x$ & $\checkmark$ \\
\hline $\begin{array}{l}\text { Ganz } \\
(2011)^{2}\end{array}$ & USA & $\begin{array}{l}5 \\
5 \\
8\end{array}$ & $\begin{array}{l}56 \\
.9\end{array}$ & $\begin{array}{l}3 \\
5\end{array}$ & - & $\begin{array}{l}6 \\
5\end{array}$ & I-II & $\begin{array}{l}\mathrm{Y} \\
\text { es }\end{array}$ & $\begin{array}{l}\text { Y } \\
\text { es }\end{array}$ & $\begin{array}{l}\mathrm{Y} \\
\text { es }\end{array}$ & $\begin{array}{l}\mathrm{Y} \\
\text { es }\end{array}$ & $\begin{array}{c}\mathrm{BCP} \\
\mathrm{T}\end{array}$ & $\begin{array}{l}\text { Po } \\
\text { st }\end{array}$ & $x$ & $x$ & $\checkmark$ \\
\hline
\end{tabular}




\begin{tabular}{|c|c|c|c|c|c|c|c|c|c|c|c|c|c|c|c|c|}
\hline $\begin{array}{l}\text { Morale } \\
\text { S } \\
(2004)^{23}\end{array}$ & $\begin{array}{c}\text { Belgi } \\
\text { um }\end{array}$ & $\begin{array}{l}1 \\
6 \\
4\end{array}$ & $\begin{array}{l}62 \\
.0\end{array}$ & - & - & $\begin{array}{l}1 \\
0 \\
0\end{array}$ & NR & $\begin{array}{l}\mathrm{N} \\
\mathrm{O}\end{array}$ & $\begin{array}{l}\mathrm{N} \\
\mathrm{O}\end{array}$ & $\begin{array}{l}\mathrm{N} \\
\mathrm{O}\end{array}$ & $\begin{array}{l}\mathrm{Y} \\
\mathrm{es}\end{array}$ & $\begin{array}{l}\text { Auth } \\
\text { or- } \\
\text { mad }\end{array}$ & $\begin{array}{c}\mathrm{Pe} \\
\text { ri }\end{array}$ & $\checkmark$ & $x$ & $\checkmark$ \\
\hline $\begin{array}{l}\text { Oleske } \\
(2004)^{22}\end{array}$ & USA & $\begin{array}{l}1 \\
2 \\
3\end{array}$ & $\begin{array}{c}58 \\
.3\end{array}$ & $\begin{array}{l}\mathrm{N} \\
\mathrm{R}\end{array}$ & $\begin{array}{l}\mathrm{N} \\
\mathrm{R}\end{array}$ & $\begin{array}{l}\mathrm{N} \\
\mathrm{R}\end{array}$ & $\begin{array}{c}\text { I- } \\
\text { VI, } \\
\text { DC } \\
\text { IS }\end{array}$ & $\begin{array}{l}\mathrm{N} \\
\mathrm{O}\end{array}$ & $\begin{array}{l}\mathrm{N} \\
\mathrm{O}\end{array}$ & $\begin{array}{l}\mathrm{N} \\
\mathrm{o}\end{array}$ & $\begin{array}{l}\mathrm{Y} \\
\text { es }\end{array}$ & $\begin{array}{c}\mathrm{BCP} \\
\mathrm{T}\end{array}$ & $\begin{array}{l}\text { Po } \\
\text { st }\end{array}$ & $\checkmark$ & $x$ & $\checkmark$ \\
\hline $\begin{array}{l}\text { Pagano } \\
(2018)^{20}\end{array}$ & Italy & $\begin{array}{l}8 \\
2\end{array}$ & $\begin{array}{c}44 \\
.0\end{array}$ & $\begin{array}{l}\mathrm{N} \\
\mathrm{R}\end{array}$ & $\begin{array}{l}\mathrm{N} \\
\mathrm{R}\end{array}$ & $\begin{array}{l}1 \\
2\end{array}$ & NR & $\begin{array}{l}\mathrm{N} \\
\mathrm{O}\end{array}$ & $\begin{array}{l}\mathrm{Y} \\
\text { es }\end{array}$ & $\begin{array}{l}\mathrm{N} \\
\mathrm{O}\end{array}$ & $\begin{array}{l}\mathrm{Y} \\
\text { es }\end{array}$ & VAS & $\begin{array}{l}\mathrm{N} \\
\mathrm{R}\end{array}$ & $\checkmark$ & $x$ & $x$ \\
\hline \multicolumn{17}{|c|}{ Cross-sectional studies } \\
\hline $\begin{array}{l}\text { Avis } \\
(2004)^{26}\end{array}$ & USA & $\begin{array}{l}2 \\
0 \\
4\end{array}$ & $\begin{array}{c}43 \\
.5\end{array}$ & $\begin{array}{l}\mathrm{N} \\
\mathrm{R}\end{array}$ & $\begin{array}{l}\mathrm{N} \\
\mathrm{R}\end{array}$ & $\begin{array}{l}\mathrm{N} \\
\mathrm{R}\end{array}$ & $\begin{array}{l}\text { I- } \\
\text { III }\end{array}$ & $\begin{array}{l}\mathrm{Y} \\
\text { es }\end{array}$ & $\begin{array}{l}\mathrm{Y} \\
\text { es }\end{array}$ & $\begin{array}{l}\mathrm{Y} \\
\mathrm{es}\end{array}$ & $\begin{array}{l}\mathrm{N} \\
\mathrm{O}\end{array}$ & $\begin{array}{c}\text { CA } \\
\text { RES }\end{array}$ & $\begin{array}{l}\text { Po } \\
\text { st }\end{array}$ & $x$ & $x$ & $\checkmark$ \\
\hline $\begin{array}{l}\text { Baumg } \\
\text { art } \\
(2011)^{25}\end{array}$ & $\begin{array}{l}\text { Swed } \\
\text { en }\end{array}$ & $\begin{array}{l}2 \\
0 \\
2\end{array}$ & $\begin{array}{c}62 \\
.7\end{array}$ & - & - & $\begin{array}{l}1 \\
0 \\
0\end{array}$ & NR & $\begin{array}{l}\mathrm{N} \\
\mathrm{O}\end{array}$ & $\begin{array}{l}\mathrm{N} \\
\mathrm{O}\end{array}$ & $\begin{array}{l}\mathrm{N} \\
\mathrm{o}\end{array}$ & $\begin{array}{l}\mathrm{Y} \\
\text { es }\end{array}$ & $\begin{array}{c}\text { UDI } \\
-6\end{array}$ & $\begin{array}{l}\text { Po } \\
\text { st }\end{array}$ & $\checkmark$ & $x$ & $\checkmark$ \\
\hline $\begin{array}{l}\text { Chen } \\
(2018)^{27}\end{array}$ & $\begin{array}{c}\text { Chin } \\
\text { a }\end{array}$ & $\begin{array}{l}6 \\
0 \\
8\end{array}$ & $\begin{array}{c}48 \\
.0\end{array}$ & $\begin{array}{l}4 \\
5\end{array}$ & - & $\begin{array}{l}5 \\
5\end{array}$ & $\begin{array}{l}0- \\
\text { IV }\end{array}$ & $\begin{array}{l}\mathrm{N} \\
\mathrm{O}\end{array}$ & $\begin{array}{l}\mathrm{Y} \\
\text { es }\end{array}$ & $\begin{array}{l}\mathrm{N} \\
\mathrm{O}\end{array}$ & $\begin{array}{l}\mathrm{N} \\
\mathrm{O}\end{array}$ & $\begin{array}{c}\text { EOR } \\
\text { TC } \\
\text { QL } \\
\text { Q- } \\
\text { C30 }\end{array}$ & $\begin{array}{l}\text { Po } \\
\text { st }\end{array}$ & $x$ & $x$ & $\checkmark$ \\
\hline $\begin{array}{l}\text { Chin } \\
(2009)^{28}\end{array}$ & $\begin{array}{c}\text { Cana } \\
\text { da }\end{array}$ & $\begin{array}{l}2 \\
5 \\
1\end{array}$ & $\begin{array}{c}60 \\
.5\end{array}$ & - & - & $\begin{array}{l}1 \\
0 \\
0\end{array}$ & NR & $\begin{array}{l}\mathrm{N} \\
\mathrm{O}\end{array}$ & $\begin{array}{l}\mathrm{N} \\
\mathrm{O}\end{array}$ & $\begin{array}{l}\mathrm{N} \\
\mathrm{O}\end{array}$ & $\begin{array}{l}\mathrm{Y} \\
\text { es }\end{array}$ & $\begin{array}{c}\text { Auth } \\
\text { or- } \\
\text { mad } \\
\text { e }\end{array}$ & $\begin{array}{c}\mathrm{Pe} \\
\text { ri }\end{array}$ & $\checkmark$ & $x$ & $\checkmark$ \\
\hline $\begin{array}{l}\text { Cook } \\
(2017)^{29}\end{array}$ & USA & $\begin{array}{l}8 \\
0 \\
0\end{array}$ & $\begin{array}{c}56 \\
.1\end{array}$ & $\begin{array}{l}1 \\
9\end{array}$ & - & $\begin{array}{l}7 \\
8\end{array}$ & NR & $\begin{array}{l}\mathrm{N} \\
\mathrm{O}\end{array}$ & $\begin{array}{l}\mathrm{N} \\
\mathrm{O}\end{array}$ & $\begin{array}{l}\mathrm{N} \\
\mathrm{O}\end{array}$ & $\begin{array}{l}\mathrm{Y} \\
\mathrm{es}\end{array}$ & NA & $\begin{array}{l}\text { Po } \\
\text { st }\end{array}$ & $\checkmark$ & $x$ & $x$ \\
\hline $\begin{array}{l}\text { Couzi } \\
(1995)^{30}\end{array}$ & USA & $\begin{array}{l}1 \\
9 \\
0\end{array}$ & $\begin{array}{c}54 \\
.9\end{array}$ & - & - & $\begin{array}{l}1 \\
0 \\
0\end{array}$ & NR & $\begin{array}{l}\mathrm{Y} \\
\text { es }\end{array}$ & $\begin{array}{l}\text { Y } \\
\text { es }\end{array}$ & $\begin{array}{l}\mathrm{Y} \\
\text { es }\end{array}$ & $\begin{array}{l}\mathrm{Y} \\
\text { es }\end{array}$ & $\begin{array}{l}\text { Auth } \\
\text { or- } \\
\text { mad }\end{array}$ & $\begin{array}{l}\text { Po } \\
\text { st }\end{array}$ & $\checkmark$ & $x$ & $\checkmark$ \\
\hline
\end{tabular}

This article is protected by copyright. All rights reserved. 


\begin{tabular}{|l|l|l|l|l|l|l|l|l|l|l|l|l|l|l|l|l|}
\hline & & & & & & & & & & & & e & & & & \\
\hline
\end{tabular}

Table 2: Characteristics of included studies (continued)

\begin{tabular}{|c|c|c|c|c|c|c|c|c|c|c|c|c|c|c|c|c|}
\hline $\begin{array}{l}\text { Danie } \\
\text { l } \\
(2014 \\
)^{31}\end{array}$ & $\begin{array}{c}\text { Engl } \\
\text { and }\end{array}$ & $\begin{array}{c}19 \\
6\end{array}$ & $\begin{array}{c}56 . \\
0\end{array}$ & $\begin{array}{l}5 \\
5\end{array}$ & $\begin{array}{l}\mathrm{N} \\
\mathrm{R}\end{array}$ & $\begin{array}{l}\mathrm{N} \\
\mathrm{R}\end{array}$ & $\begin{array}{l}\mathrm{N} \\
\mathrm{R}\end{array}$ & $\begin{array}{c}\mathrm{N} \\
\mathrm{O}\end{array}$ & $\begin{array}{l}\mathrm{Y} \\
\text { es }\end{array}$ & $\begin{array}{l}N \\
0\end{array}$ & $\begin{array}{l}\mathrm{Y} \\
\mathrm{es}\end{array}$ & MRS & $\begin{array}{l}\mathrm{N} \\
\mathrm{R}\end{array}$ & $\checkmark$ & $x$ & $\checkmark$ \\
\hline $\begin{array}{l}\text { Ganz } \\
(2003 \\
)^{32}\end{array}$ & USA & $\begin{array}{c}57 \\
7\end{array}$ & $\begin{array}{c}49 . \\
5\end{array}$ & $\begin{array}{l}1 \\
6\end{array}$ & $\begin{array}{l}1 \\
3\end{array}$ & $\begin{array}{l}6 \\
0\end{array}$ & $\begin{array}{l}0- \\
\text { II }\end{array}$ & $\begin{array}{l}\mathrm{Y} \\
\mathrm{es}\end{array}$ & $\begin{array}{l}\mathrm{Y} \\
\text { es }\end{array}$ & $\begin{array}{l}\mathrm{N} \\
\mathrm{O}\end{array}$ & $\begin{array}{l}\mathrm{Y} \\
\mathrm{es}\end{array}$ & $\begin{array}{c}\mathrm{BCP} \\
\mathrm{T}\end{array}$ & $\begin{array}{l}\text { Po } \\
\text { st }\end{array}$ & $\checkmark$ & $x$ & $x$ \\
\hline $\begin{array}{l}\text { Green } \\
\text { dale } \\
(2001 \\
)^{33}\end{array}$ & USA & 61 & $\begin{array}{c}54 . \\
5\end{array}$ & - & - & $\begin{array}{l}1 \\
0 \\
0\end{array}$ & $\begin{array}{l}\text { I- } \\
\text { II }\end{array}$ & $\begin{array}{l}\mathrm{Y} \\
\mathrm{es}\end{array}$ & $\begin{array}{l}\mathrm{Y} \\
\text { es }\end{array}$ & $\begin{array}{l}\mathrm{N} \\
\mathrm{O}\end{array}$ & $\begin{array}{l}\mathrm{Y} \\
\text { es }\end{array}$ & $\begin{array}{c}\mathrm{BCP} \\
\mathrm{T}\end{array}$ & $\begin{array}{c}\text { Po } \\
\text { st }\end{array}$ & $x$ & $x$ & $\checkmark$ \\
\hline $\begin{array}{l}\text { Gupta } \\
(2006 \\
)^{34}\end{array}$ & UK & $\begin{array}{c}20 \\
0\end{array}$ & $\begin{array}{c}53 . \\
9\end{array}$ & 6 & 9 & $\begin{array}{l}6 \\
6\end{array}$ & $\begin{array}{l}\mathrm{N} \\
\mathrm{R}\end{array}$ & $\begin{array}{l}\mathrm{Y} \\
\text { es }\end{array}$ & $\begin{array}{l}\mathrm{Y} \\
\text { es }\end{array}$ & $\begin{array}{l}\mathrm{Y} \\
\text { es }\end{array}$ & $\begin{array}{c}\mathrm{Y} \\
\text { es }\end{array}$ & MRS & $\begin{array}{c}\text { Po } \\
\text { st }\end{array}$ & $\checkmark$ & $x$ & $x$ \\
\hline $\begin{array}{l}\text { Landi } \\
(2016 \\
)^{35}\end{array}$ & USA & $\begin{array}{c}54 \\
8\end{array}$ & $\begin{array}{c}58 . \\
1\end{array}$ & - & - & $\begin{array}{l}1 \\
0 \\
0\end{array}$ & $\begin{array}{c}0- \\
\text { II } \\
\text { I }\end{array}$ & $\begin{array}{l}\mathrm{N} \\
\mathrm{O}\end{array}$ & $\begin{array}{l}\mathrm{Y} \\
\text { es }\end{array}$ & $\begin{array}{l}\mathrm{N} \\
\mathrm{O}\end{array}$ & $\begin{array}{c}\mathrm{Y} \\
\text { es }\end{array}$ & $\begin{array}{c}\text { Interv } \\
\text { iew }\end{array}$ & $\begin{array}{l}\text { Po } \\
\text { st }\end{array}$ & $\checkmark$ & $x$ & $x$ \\
\hline $\begin{array}{l}\text { Leini } \\
\text { ng } \\
(2006 \\
)^{36}\end{array}$ & USA & $\begin{array}{c}37 \\
1\end{array}$ & $\begin{array}{c}36 . \\
2\end{array}$ & $\begin{array}{l}1 \\
0 \\
0\end{array}$ & - & - & $\begin{array}{c}0- \\
\text { II } \\
\text { I }\end{array}$ & $\begin{array}{l}\mathrm{Y} \\
\mathrm{es}\end{array}$ & $\begin{array}{l}\mathrm{Y} \\
\text { es }\end{array}$ & $\begin{array}{l}\mathrm{Y} \\
\text { es }\end{array}$ & $\begin{array}{l}\mathrm{Y} \\
\mathrm{es}\end{array}$ & $\begin{array}{c}\mathrm{BCP} \\
\mathrm{T}\end{array}$ & $\begin{array}{c}\text { Po } \\
\text { st }\end{array}$ & $\checkmark$ & $x$ & $x$ \\
\hline $\begin{array}{l}\text { Morro } \\
\text { W } \\
(2014 \\
)^{37}\end{array}$ & USA & $\begin{array}{l}1,0 \\
90\end{array}$ & $\begin{array}{c}47 . \\
6\end{array}$ & $\begin{array}{l}8 \\
2\end{array}$ & 1 & $\begin{array}{l}1 \\
3\end{array}$ & $\begin{array}{l}\mathrm{I}- \\
\mathrm{I} \\
\mathrm{V}\end{array}$ & $\begin{array}{l}\mathrm{Y} \\
\text { es }\end{array}$ & $\begin{array}{l}\mathrm{Y} \\
\text { es }\end{array}$ & $\begin{array}{l}\mathrm{N} \\
\mathrm{O}\end{array}$ & $\begin{array}{c}\mathrm{Y} \\
\mathrm{es}\end{array}$ & $\begin{array}{c}\mathrm{BCP} \\
\mathrm{T}\end{array}$ & $\begin{array}{l}\text { Po } \\
\text { st }\end{array}$ & $x$ & $x$ & $\checkmark$ \\
\hline $\begin{array}{l}\text { Park } \\
(2013 \\
)^{38}\end{array}$ & $\begin{array}{c}\text { Kore } \\
\text { a }\end{array}$ & $\begin{array}{c}20 \\
0\end{array}$ & $\begin{array}{c}45 . \\
6\end{array}$ & $\begin{array}{l}6 \\
4\end{array}$ & $\begin{array}{l}1 \\
6\end{array}$ & $\begin{array}{l}2 \\
0\end{array}$ & $\begin{array}{l}\text { I- } \\
\text { I } \\
\text { V }\end{array}$ & $\begin{array}{l}\mathrm{Y} \\
\text { es }\end{array}$ & $\begin{array}{l}\mathrm{Y} \\
\text { es }\end{array}$ & $\begin{array}{l}N \\
0\end{array}$ & $\begin{array}{c}N \\
0\end{array}$ & MRS & $\begin{array}{l}\mathrm{N} \\
\mathrm{R}\end{array}$ & $x$ & $x$ & $\checkmark$ \\
\hline
\end{tabular}

This article is protected by copyright. All rights reserved. 


\begin{tabular}{|c|c|c|c|c|c|c|c|c|c|c|c|c|c|c|c|c|}
\hline $\begin{array}{l}\text { Robin } \\
\text { son } \\
(2017 \\
)^{24}\end{array}$ & $\begin{array}{c}\text { Austr } \\
\text { alia }\end{array}$ & $\begin{array}{c}62 \\
5\end{array}$ & $\begin{array}{c}65 . \\
1\end{array}$ & $\begin{array}{l}\mathrm{N} \\
\mathrm{R}\end{array}$ & $\begin{array}{l}\mathrm{N} \\
\mathrm{R}\end{array}$ & $\begin{array}{l}\mathrm{N} \\
\mathrm{R}\end{array}$ & I & $\begin{array}{l}\mathrm{Y} \\
\mathrm{es}\end{array}$ & $\begin{array}{l}\mathrm{Y} \\
\text { es }\end{array}$ & $\begin{array}{l}Y \\
\text { es }\end{array}$ & $\begin{array}{c}\mathrm{Y} \\
\text { es }\end{array}$ & $\begin{array}{c}\text { QUI } \\
\text { D }\end{array}$ & $\begin{array}{l}\text { Po } \\
\text { st }\end{array}$ & $\checkmark$ & $x$ & $x$ \\
\hline $\begin{array}{l}\text { Schov } \\
\text { er } \\
(2014 \\
)^{39}\end{array}$ & USA & $\begin{array}{c}12 \\
9\end{array}$ & $\begin{array}{c}64 . \\
0\end{array}$ & $\begin{array}{l}\mathrm{N} \\
\mathrm{R}\end{array}$ & $\begin{array}{l}\mathrm{N} \\
\mathrm{R}\end{array}$ & $\begin{array}{l}\mathrm{N} \\
\mathrm{R}\end{array}$ & $\begin{array}{l}\text { I- } \\
\text { II }\end{array}$ & $\begin{array}{l}\mathrm{Y} \\
\text { es }\end{array}$ & $\begin{array}{l}\mathrm{Y} \\
\text { es }\end{array}$ & $\begin{array}{l}\mathrm{N} \\
\mathrm{O}\end{array}$ & $\begin{array}{l}\mathrm{N} \\
\mathrm{O}\end{array}$ & BESS & $\begin{array}{l}\text { Po } \\
\text { st }\end{array}$ & $x$ & $x$ & $\checkmark$ \\
\hline $\begin{array}{l}\text { Stanto } \\
\mathrm{n} \\
(2005 \\
)^{40}\end{array}$ & USA & $\begin{array}{r}2,2 \\
08\end{array}$ & $\begin{array}{c}47 . \\
0- \\
57 . \\
0^{\gamma}\end{array}$ & $\begin{array}{l}\mathrm{N} \\
\mathrm{R}\end{array}$ & $\begin{array}{l}\mathrm{N} \\
\mathrm{R}\end{array}$ & $\begin{array}{l}\mathrm{N} \\
\mathrm{R}\end{array}$ & $\begin{array}{l}0- \\
\text { II } \\
\mathrm{I}^{\delta}\end{array}$ & $\begin{array}{l}\mathrm{Y} \\
\mathrm{es}\end{array}$ & $\begin{array}{l}\mathrm{Y} \\
\text { es }\end{array}$ & $\begin{array}{l}\mathrm{N} \\
\mathrm{O}\end{array}$ & $\begin{array}{l}\mathrm{Y} \\
\text { es }\end{array}$ & $\begin{array}{c}\mathrm{BCP} \\
\mathrm{T}\end{array}$ & $\begin{array}{l}\text { Po } \\
\text { st }\end{array}$ & $x$ & $x$ & $\checkmark$ \\
\hline
\end{tabular}

$\alpha$ : Authors provided proportions of women in age range.

$ß$ : Authors provided proportions of women in age range.

$\gamma$ : Authors provided mean age for each sample. Sample 1: 56, Sample 2: 50, Sample 3: 57.

$\delta$ : Authors provided staging for each group. Sample 1: 0-II; Sample 2: 0-II; Sample 3: I-II.

NR, not reported; DCIS, ductal carcinoma in situ; Chemo, chemotherapy; Radio, radiation therapy; Endo, endocrine therapy; EORTC QLQ-C30, European Organization for the Research and Treatment of Cancer Quality of Life Questionnaire; MRS, Menopause Rating Scale; MSS, Menopausal Symptom Scale; BCPT, Breast Cancer Prevention Trial; CARES, Cancer Rehabilitation Evaluation System; FACT, Functional Assessment of Cancer Therapy; BFLUTS, Bristol Female 
Lower Urinary Tract Symptoms questionnaire; GCS, Greene Climacteric Scale; QUID, Questionnaire for Urinary Incontinence Diagnosis; VAS, Visual Analog Scale.

This article is protected by copyright. All rights reserved. 


\section{University Library}

\section{- M I N E R VA \\ A gateway to Melbourne's research publications}

Minerva Access is the Institutional Repository of The University of Melbourne

Author/s:

Colombage, UN;Lin, K-Y;Soh, S-E;Frawley, HC

Title:

Prevalence and impact of bladder and bowel disorders in women with breast cancer: A systematic review with meta-analysis

Date:

2020-10-05

\section{Citation:}

Colombage, U. N., Lin, K. -Y., Soh, S. -E. \& Frawley, H. C. (2020). Prevalence and impact of bladder and bowel disorders in women with breast cancer: A systematic review with meta-analysis. NEUROUROLOGY AND URODYNAMICS, 40 (1), pp.15-27. https:// doi.org/10.1002/nau.24531.

Persistent Link:

http://hdl.handle.net/11343/276403 